\title{
Exploring targets of TET2-mediated methylation reprogramming as potential discriminators of prostate cancer progression
}

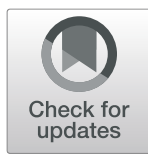

Shivani Kamdar ${ }^{1,2}$, Ruth Isserlin ${ }^{3}$, Theodorus Van der Kwast ${ }^{2,4}$, Alexandre R. Zlotta ${ }^{1,5}$, Gary D. Bader ${ }^{3}$, Neil E. Fleshner ${ }^{5}$ and Bharati Bapat ${ }^{1,2,45^{*}}$ (D)

\begin{abstract}
Background: Global DNA methylation alterations are hallmarks of cancer. The tumor-suppressive TET enzymes, which are involved in DNA demethylation, are decreased in prostate cancer (PCa); in particular, TET2 is specifically targeted by androgen-dependent mechanisms of repression in PCa and may play a central role in carcinogenesis. Thus, the identification of key genes targeted by TET2 dysregulation may provide further insight into cancer biology.

Results: Using a CRISPR/Cas9-derived TET2-knockout prostate cell line, and through whole-transcriptome and wholemethylome sequencing, we identified seven candidate genes-ASB2, ETNK2, MEIS2, NRG1, NTN1, NUDT10, and SRPX — exhibiting reduced expression and increased promoter methylation, a pattern characteristic of tumor suppressors. Decreased expression of these genes significantly discriminates between recurrent and non-recurrent prostate tumors from the Cancer Genome Atlas (TCGA) cohort $(n=423)$, and ASB2, NUDT10, and SRPX were significantly correlated with lower recurrence-free survival in patients by Kaplan-Meier analysis. ASB2, MEIS2, and SRPX also showed significantly lower expression in high-risk Gleason score 8 tumors as compared to low or intermediate risk tumors, suggesting that these genes may be particularly useful as indicators of PCa progression. Furthermore, methylation array probes in the TCGA dataset, which were proximal to the highly conserved, differentially methylated sites identified in our TET2-knockout cells, were able to significantly distinguish between matched prostate tumor and normal prostate tissues ( $n=50$ pairs). Except ASB2, all genes exhibited significantly increased methylation at these probes, and methylation status of at least one probe for each of these genes showed association with measures of PCa progression such as recurrence, stage, or Gleason score. Since ASB2 did not have any probes within the TET2-knockout differentially methylated region, we validated ASB2 methylation in an independent series of matched tumor-normal samples $(n=19)$ by methylation-specific $\mathrm{QPCR}$, which revealed concordant and significant increases in promoter methylation within the TET2-knockout site.

Conclusions: Our study identifies seven genes governed by TET2 loss in PCa which exhibit an association between their methylation and expression status and measures of PCa progression. As differential methylation profiles and TET2 expression are associated with advanced PCa, further investigation of these specialized TET2 targets may provide important insights into patterns of carcinogenic gene dysregulation.
\end{abstract}

Keywords: Prostate cancer, TET2 knockout, Differential methylation profiling, Integrative analysis, Tumor progression

\footnotetext{
* Correspondence: bapat@lunenfeld.ca

${ }^{1}$ Lunenfeld-Tanenbaum Research Institute, Mount Sinai Hospital, 60 Murray

Street, L6-304B, Toronto, ON M5T 3L9, Canada

${ }^{2}$ Department of Laboratory Medicine and Pathobiology, University of

Toronto, Medical Sciences Building (6th floor), 1 King's College Circle,

Toronto, ON M5S 1A8, Canada

Full list of author information is available at the end of the article
}

(c) The Author(s). 2019 Open Access This article is distributed under the terms of the Creative Commons Attribution 4.0 International License (http://creativecommons.org/licenses/by/4.0/), which permits unrestricted use, distribution, and reproduction in any medium, provided you give appropriate credit to the original author(s) and the source, provide a link to the Creative Commons license, and indicate if changes were made. The Creative Commons Public Domain Dedication waiver (http://creativecommons.org/publicdomain/zero/1.0/) applies to the data made available in this article, unless otherwise stated. 


\section{Introduction}

Prostate cancer (PCa) is the second most common cancer diagnosed in men worldwide, with significant global increases in incidence rates (2.4-21.4\%) in 23 countries over the last 10 years $[1,2]$. Widespread epigenomic dysregulation events in PCa have been identified as a hallmark of tumorigenesis [3-6]. Among these, tumor-specific DNA methylation $(5 \mathrm{mC})$ alterations and repression of gene expression are an emerging class of biomarkers as well as potential therapeutic targets, highlighting their importance in prostate carcinogenesis [7-14].

In PCa specifically, differential genomic patterns of $5 \mathrm{mC}$ and its derivative, 5 -hydroxymethylcytosine $(5 \mathrm{hmC})$ have been linked to distinct molecular subtypes, indicating clear epigenetic stratification within tumors. For example, TMPRSS2-ERG fusion-positive tumors and tumors showing either SPOP or FOXA1 mutations possess distinct methylation signatures as identified by unsupervised clustering [5], while significant loss of $5 \mathrm{hmC}$ is observed only within ERG-fusion negative PCa [15]. Our previous studies have shown that locus-specific $5 \mathrm{hmC}$ alteration is significantly correlated to transcriptional repression of multiple genes in prostate cancer cell lines, indicating the potential functional importance of these changes [16].In normal cells, the ten-eleven translocase (TET) family of genes oxidize $5 \mathrm{mC}$ to $5 \mathrm{hmC}$, regulating levels of both epigenetic marks and promoting demethylation. The TET family consists of three members-TET1, TET2, and TET3-which generate $5 \mathrm{hmC}$ through $\mathrm{Fe}^{2+}$ and $\alpha$-ketoglutarate-dependent dioxygenase activity [17, 18]. While all TET genes show loss of expression in PCa tissues, their mutation frequencies in primary prostate tumors are low, in contrast to the high mutation rates observed in metastatic castration-resistant prostate cancer and hematological malignancies [19-24].

Of the three TET enzymes, TET2 in particular is uniquely implicated as having a central role in PCa biology due to its key involvement with androgen receptor (AR) signaling. TET2 is able to bind to AR and its transcriptional coactivators in a prostate-specific interaction and has been linked to regulation of androgen-dependent genes such as PSA [23]. In turn, expression of the AR-induced microRNAs 29a and 29b specifically target and downregulate TET2 in $\mathrm{PCa}$, resulting in activation of $\mathrm{AR}$ and mTOR signaling pathways and promoting pro-carcinogenic biological functions $[19,20]$. In contrast, its family member TET3 has not been well investigated in the context of PCa and, although TET1 is known to be co-recruited along AR as part of a hormonal response in normal prostate cells, this link has not yet been investigated in PCa [25]. Furthermore, loss of TET2 activity has profound implications on $\mathrm{PCa}$ development, where reduced TET2 expression is correlated with decreased disease-free survival, increased Gleason score, and metastasis [20, 23]. Thus, there is ample evidence to suggest that TET2 loss may act as a key mechanism of $\mathrm{PCa}$ development, and exploration of its downstream target genes may provide new insights into cancer biology.

To investigate TET2's role in $\mathrm{PCa}$ pathogenesis, we generated TET2 knockout (KO) cell-lines in representative normal prostate cells to discover key candidate genes regulated by TET2-mediated methylation reprogramming. Here, we describe seven promising targets of epigenetic modification directed by TET2 loss by analyzing their methylation and expression profiles in both prostate-derived cell lines and prostate tumors from the Cancer Genome Atlas (TCGA). These target genes are able to significantly differentiate between recurrent and non-recurrent tumors based on their expression status, further implicating TET2-governed changes as a significant process in carcinogenesis. Promoter methylation gain of one such promising candidate gene, ankyrin repeat and SOCS-box containing protein 2 (ASB2), within the TET2-target site is investigated in an independent series of primary prostate tumors. These studies elucidate the dynamics of TET2-mediated gene regulation in order to develop a novel selection strategy for identifying genes whose methylation and differential expression may play a key role in prostate carcinogenesis.

\section{Results}

\section{Generation of TET2-knockout prostate cell lines}

Sanger sequencing of clonally expanded populations using primers surrounding the indel target region (Additional file 1: Figure S1; Additional file 2: Table S1) revealed two CRISPR-knockout clones (CR1 and CR2; derived from the "P38" Sigma gRNA sequence [see the "Materials and methods" section for details]) exhibiting truncation mutations resulting in loss of both binding and catalytic domains [26]. CR1 showed heterozygous deletion of a single A nucleotide on one allele, resulting in a premature stop codon at 291aa, while CR2 showed a 17-bp deletion on one strand and a 2-bp deletion on another, resulting in functional homozygous knockout and stop codons present at 252aa or 256aa, respectively (Fig. 1a). Additional primer sequences were assessed to confirm that no off-target effects were observed (Additional file 2: Table S1, Additional file 3: Figure S2) as per the protocol described by Mali et al. [27]. Briefly, the last 13 bases of the gRNA sequence, along with the TGG protospacer sequence, were run through NCBI BLAST, and sequences with the highest similarity and including the protospacer sequence were assessed. The two sites tested for off-target effects possessed $93.8 \%$ sequence similarity $(15 / 16$ bases matching) to the BLASTed sequence. Complete loss of TET2 protein expression in knockouts versus parental cells was 
confirmed via Western blot (Fig. 1b, Additional file 4: Figure S3).

\section{Methylation and expression profiles show extensive epigenetic silencing in TET2-knockout cells}

We performed next-generation sequencing (NGS) of genomic DNA following methyl-binding protein capture (MBD-Seq) and poly-A enriched mRNA (RNA-seq) to determine the effects of TET2 loss on prostate cells at both whole-methylome and whole-transcriptome levels. We observed large increases in differentially methylated regions (DMRs) in KOs versus parental cells (Fig. 1c, Additional file 5: Figure S4). Similarly, extensive changes in gene expression were observed in both KOs, with $17.3 \%$ and $4.5 \%$ more genes showing significant downregulation $(>1.5$-fold decrease, $p<0.05)$ than upregulation ( $>1.5$-fold increase, $p<0.05)$ in CR1 and CR2, respectively (Fig. 1d, Additional file 6: Figure S5). 47.1\% of downregulated genes and $42.3 \%$ of upregulated genes were shared between both TET2 KO cell lines.

As promoter methylation gain is likely correlated with decreases in gene expression in cancer, especially in tumor suppressors [15-17], we examined the proportion of genes that showed both significant downregulation of expression $(p<0.05$, edge $\mathrm{v}$ 3.8.6) and concomitant increased promoter methylation $(p<0.05$, DiffBind), in either knockout (Fig. 1e), of which $36.7 \%$ were commonly shared between both KOs. We found that 14.5\% (146 genes) of all genes exhibiting promoter methylation in CR1 also showed significant expression loss, which was comparable to the $16.6 \%$ of such genes (291 genes) discovered in CR2. A further 54 of these genes overlapped between both knockouts (Additional file 7: Table S2).

Subsequently, all genes exhibiting expression changes in either knockout were further assessed to determine their suitability as key targets of TET2-mediated reprogramming.

\section{A selected panel of key candidate genes exhibits differential methylation and downregulation in an independent cohort of primary prostate tumors}

To identify the most significantly affected TET2-target genes with evidence of the highest potential impact in our model, we first compared global expression profiles in our cell line models to those reported in a subset of tumors from the TCGA which were in the bottom 10th percentile of TET2 expression ( $n=43$ of 423 total tumors) as compared to normal prostate tissue from the same cohort $(n=35)$. Out of 4192 genes with significantly decreased expression in either TET2 knockout $(p<0.05, \mathrm{FC}<0.75$, edgeR; independent of methylation status), 780 genes exhibited significant loss of expression below the Bonferroni-corrected $p$ value threshold of 1.193E-5 (Mann-Whitney $U$ test) in the low-TET2 TCGA subset (Fig. 2a, Additional file 8: Figure S6). Of these genes, 61 exhibited significant promoter methylation $(p<0.05$, DiffBind) in our knockout cell lines, all of which had also been identified as exhibiting significant $5 \mathrm{hmC}$ loss in cancer cells from our previous study [16], implicating them as strong potential targets of TET-mediated demethylation (Additional file 9: Table S3). In turn, all but one of these genes exhibited significantly altered methylation in the low-TET2 TCGA tumors with methylation array data available $(n=43)$ as compared to normal prostate samples $(n=50)$. Fourteen of these 60 genes exhibited significant association with ERG fusion status (Mann-Whitney $U$ test, Bonferroni-corrected $p$-value $<0.05)$, of which 11 showed significantly increased expression in ERG-positive tumors and three showed significantly decreased expression (Additional file 10: Table S4). TET2 expression was also significantly decreased in ERG-negative as compared to ERG-positive tumors $(p=7.124 \mathrm{E}-05$, Mann-Whitney $U$ test).

Next, we assessed the ability of these 60 TET2-target genes to distinguish between prostate tumor and normal samples $(n=35)$ expressing variable levels of TET2, in either all tumors with expression data available $(n=423$, Fig. 2b) or in matched tumor and normal pairs only ( $n$ $=35$, Fig. 2c). Twenty-seven genes exhibited significant loss of expression below the Bonferroni-corrected $p$ value threshold $(p<4.2373 \mathrm{E}-4$, Mann-Whitney $U$ test) in both sets and were further assessed via pathway analysis.

\section{TET2-target genes are involved in critical biological processes governing signaling interactions and the immune system}

We assessed significantly enriched biological pathway annotations (binomial $p$ value $<0.05$ ) for (a) all methylated and downregulated genes from our TET2-KO cells alone and (b) the 27 candidate genes identified above, with the Genomic Regions Enrichment of Annotations Tool (GREAT), using the whole genome as a background. Enriched pathways would not only highlight key functions significantly affected by global TET2-mediated alteration of the genes (set a), but would also provide insight into the potential biological consequences of candidate gene silencing (set $b$ ).

Among all silenced genes in our knockouts (set a), many of the pathways enriched were associated with immune functionality, cellular adhesion, and cell death, especially with regards to the formation of inflammasomes, oligomeric complexes involved in inflammatory cytokine generation, and cell death (Additional file 11: Figure S7). When examining the panel of 27 candidate genes (set b), a differential pathway clustering profile enriched for pathways including phosphate metabolism, microtubule organization, cell-cell signaling, and DNA helicase activity was observed (Fig. 3). Interestingly, five of the 27 


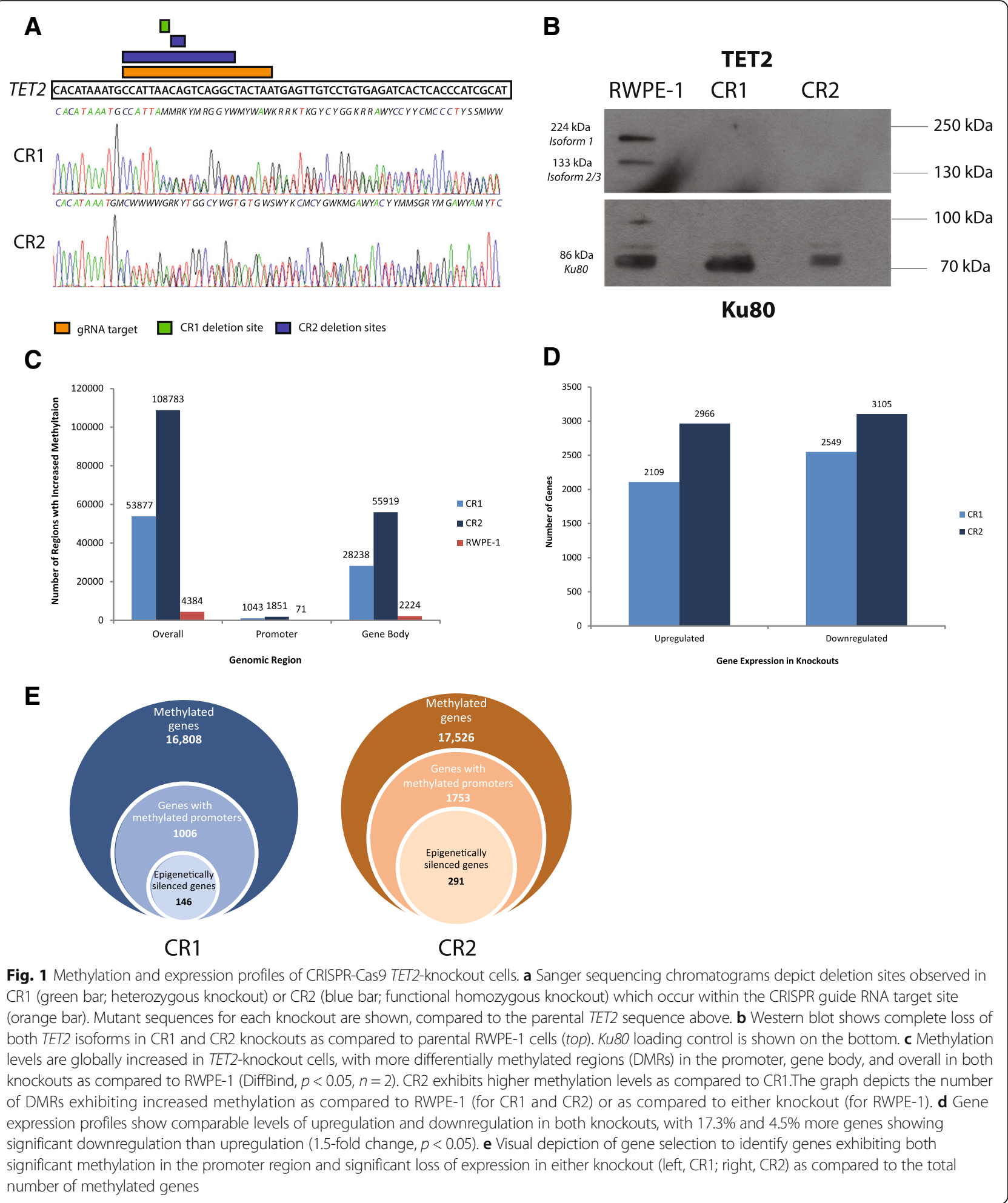

candidate genes identified above (ANO1, MEIS2, PDE4A, $P M P 22$, and $S R P X)$ were identified through pathway analysis as genes known to be downregulated in prostate cancer samples specifically, indicating the relevance of these candidates to $\mathrm{PCa}$ in other datasets as well.
Expression levels and methylation status of key TET2-target genes exhibit correlation with measures of prostate cancer progression

As reduced TET2 levels are correlated with advanced PCa and decreased survival $[19,22]$, we assessed the 
A
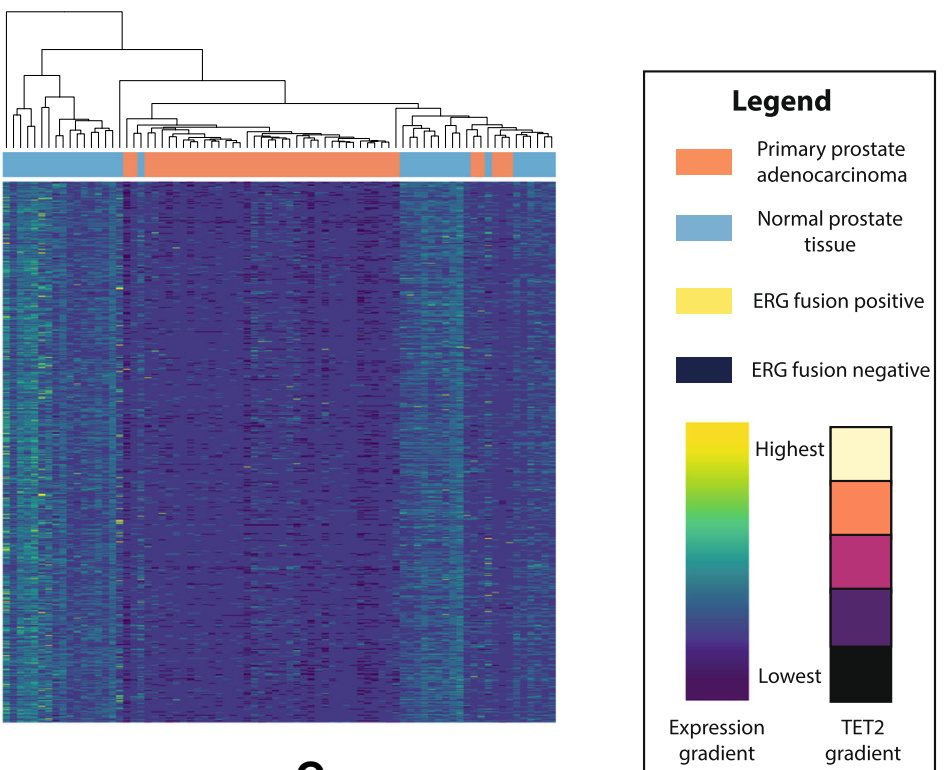

B
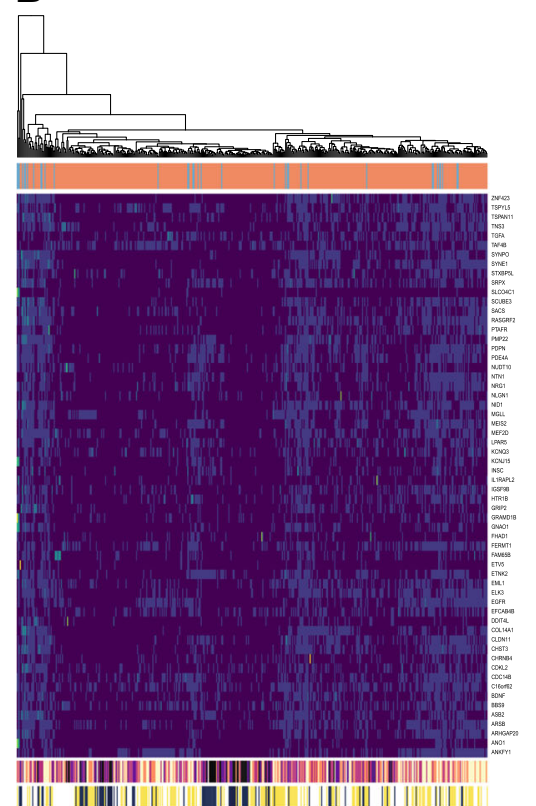

C

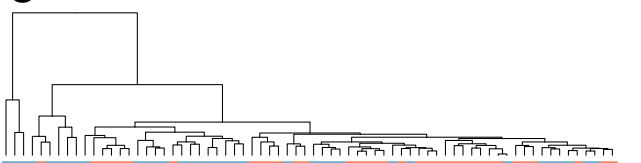

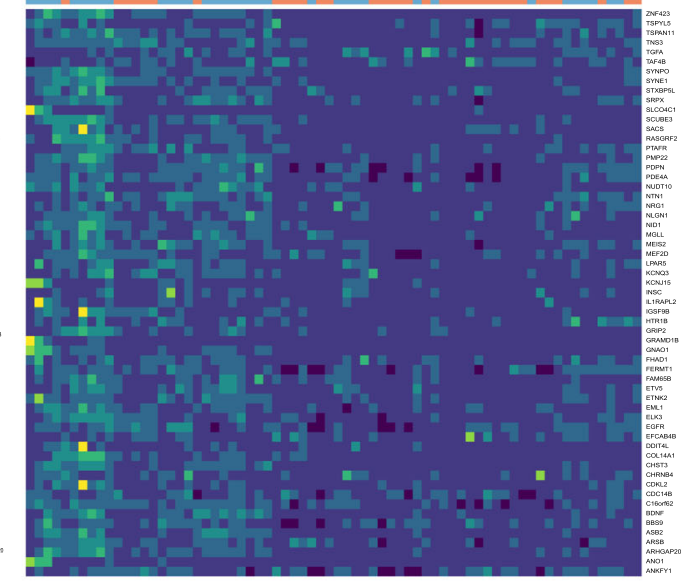

Fig. 2 Genes exhibiting expression loss in TET2-knockout cell lines show discriminatory ability between normal prostate and prostate tumor based on expression status. Unsupervised heatmaps depict expression values normalized by gene for a 780 genes exhibiting significant loss of expression in both TET2-knockout cells (edgeR, $p<0.05$ ) and a subset of tumors from the Cancer Genome Atlas (TCGA) within the lowest 10th percentile of TET2 expression (Mann-Whitney U, $p<1.193 \mathrm{E}-5$ ), on this low-TET2 tumor subset; $\mathbf{b} 60$ genes matching the above criteria and exhibiting increased promoter methylation in TET2 knockouts (DiffBind, $p<0.05)$, in all TCGA tumors with expression data available $(n=423)$ or c in matched tumor and normal pairs $(n=35)$. Expression gradient bar indicates normalized expression levels, ranging from highest (yellow) to lowest (dark blue). TET2 gradient bar indicates TET2 expression in the entire TCGA dataset, ranging from highest (cream) to lowest (black). ERG fusion status is annotated in the entire TCGA dataset where data is available. Dendrograms indicate clustering between tissue samples

ability of changes in expression of our 27 TET2-target candidate genes to discriminate based on pathological stage, Gleason score (GS), and recurrence in the TCGA dataset ( $n=423$ tumor samples). Lowered expression of nine of these genes was able to significantly distinguish recurrent tumors from non-recurrent tumors (Mann-Whitney $U$ test, $p<0.05$, Table 1 ). Five genes could distinguish between tumors of differing pathological stage and Gleason scores. Of these, ankyrin repeat and SOCS box protein 2 (ASB2), Meis homeobox 2 


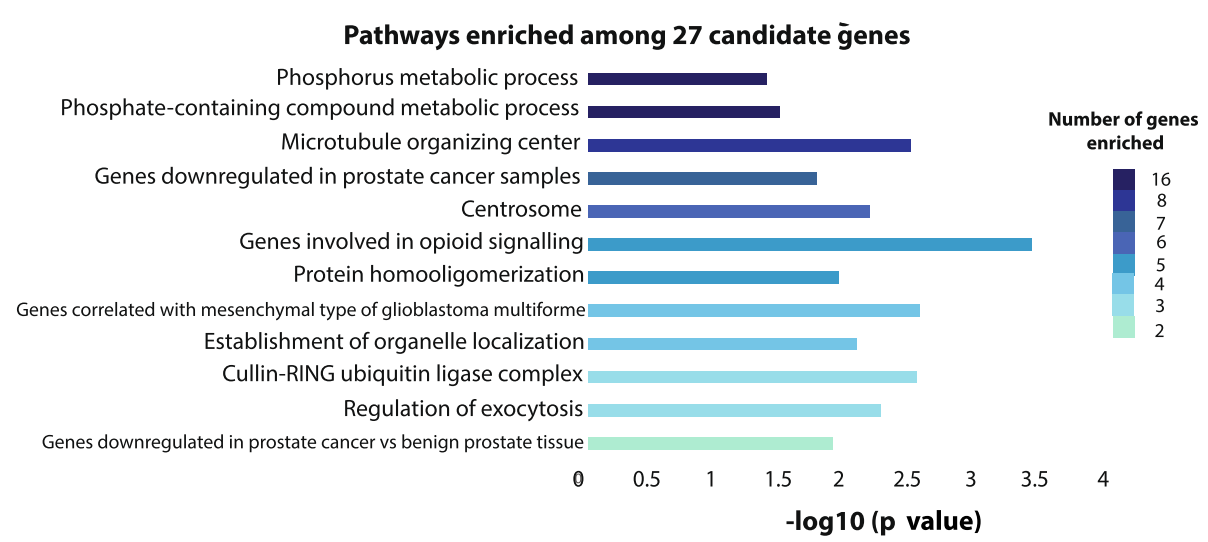

Fig. 3 Pathway analysis of candidate genes significantly altered by TET2-knockout. Selected, significant pathway enrichment annotations from GREAT for genes exhibiting significantly altered expression in both TET2-knockout cell lines and in tumor versus normal samples from the TCGA (binomial $p$ value $<0.05$ ). Bar coloration indicates the number of candidate genes enriched within each pathway

(MEIS2), and sushi repeat-containing protein X-linked $(S R P X)$ were able to specifically distinguish GS6 or GS7 from GS8 or higher tumors (Bonferroni-adjusted Dunn test, $p<0.05$ ).

We next examined the performance of these nine genes' expression levels in discriminating between prostate tumor and normal tissue through receiver operating curve (ROC) analysis in the TCGA cohort (Fig. 4a, Table 2). All genes exhibited strong performance, with ETNK2 expression providing the highest accuracy of classification (AUC $=0.919,95 \%$ CI $0.886-0.952$; sensitivity $=0.797$, specificity $=0.943$ ). However, although all nine genes were significant predictors of tumor status in univariate logistic regression analyses, four genes in particular-MEIS2, NRG1, NTN1, and NUDT10-were determined to be independent predictors by multivariate logistic regression (Table 3 ).

As Mann-Whitney $U$ analysis indicated that the expression of the nine candidate genes was correlated with recurrence, we assessed whether decreased gene expression was correlated with recurrence-free survival in $\mathrm{PCa}$ through $\mathrm{X}$-tile and Kaplan-Meier analysis. Lowered expression of three of the nine genes-ASB2, $N U D T 10$, and $S R P X$-was significantly correlated with poor prognosis in PCa patients (Fig. 4b), while two more genes-ETNK2 and NTN1-were trending $(p<0.10$, Additional file 12: Figure S8). Overall, these analyses exhibited the utility of these genes as promising indicators of $\mathrm{PCa}$ status and progression.

We subsequently examined methylation of the nine candidate genes able to distinguish recurrent tumors in the TCGA database at sites proximal to $( \pm 500 \mathrm{bp})$ or within regions gaining methylation in our $\mathrm{KO}$ cell line models to assess possible linkages between the TET2target gene panel and PCa. Out of the nine genes, seven possessed TCGA probes proximal to our TET2-KO differential methylation sites which showed discriminatory ability between matched tumor and normal samples $(n=50$ pairs) based on differential methylation values at specific CpG sites (Table 4, Fig. 5a).

Table 1 Significant changes in TET2-target gene expression associated with prostate cancer development and progression

\begin{tabular}{|c|c|c|c|c|c|c|c|c|}
\hline Gene & $\begin{array}{l}\text { Tumor vs normal }{ }^{1} \\
\text { Matched tumor } \\
\text { and normal }\end{array}$ & $\begin{array}{l}\text { Tumor vs normal } \\
\text { All TCGA tumors }\end{array}$ & Recurrence $^{1}$ & Stage $^{1}$ & Gleason score (overall) $)^{2}$ & GS6 vs GS7 ${ }^{3}$ & GS6 vs GS8 $+^{3}$ & GS7 vs GS8 $+{ }^{3}$ \\
\hline ASB2 & $2.9543 \mathrm{E}-08$ & $\underline{1.1163 \mathrm{E}-10}$ & $\underline{0.0189}$ & $\underline{0.0095}$ & $\underline{3.1843 \mathrm{E}-05}$ & 0.17 & $\underline{0.00044413}$ & $2.1003 \mathrm{E}-4$ \\
\hline ETNK2 & $\underline{8.0425 \mathrm{E}-15}$ & $\underline{1.6991 \mathrm{E}-16}$ & $\underline{0.0147}$ & 0.3725 & 0.1526 & 1 & 0.4005 & 0.0924 \\
\hline KCNJ15 & $1.1797 \mathrm{E}-09$ & $\underline{6.9856 \mathrm{E}-14}$ & $\underline{0.0436}$ & 0.6534 & 0.0842 & 0.4247 & 1 & $\underline{0.0487}$ \\
\hline MEIS2 & 8.6435E-09 & $\underline{1.4128 \mathrm{E}-13}$ & $\underline{0.0125}$ & $\underline{0.0256}$ & $\underline{0.0010}$ & 0.5208 & $\underline{0.0100}$ & $\underline{0.0015}$ \\
\hline NRG1 & $\underline{1.6456 \mathrm{E}-05}$ & $1.9533 \mathrm{E}-11$ & $\underline{0.0013}$ & $\underline{0.0023}$ & $\underline{0.0016}$ & 1 & 0.0918 & $6.6543 \mathrm{E}-4$ \\
\hline NTN1 & $\underline{3.1531 \mathrm{E}-05}$ & $\underline{1.1782 \mathrm{E}-07}$ & $\underline{0.0091}$ & $\underline{0.0261}$ & $\underline{0.0273}$ & 1 & 0.205 & $\underline{0.0139}$ \\
\hline NUDT10 & $2.4896 \mathrm{E}-08$ & $2.8735 \mathrm{E}-10$ & 0.0235 & 0.5739 & 0.4542 & 0.4667 & 0.3151 & 0.9259 \\
\hline PDE4A & $\underline{2.1250 \mathrm{E}-09}$ & $\underline{1.2586 \mathrm{E}-12}$ & $\underline{0.0431}$ & 0.6292 & 0.5444 & 0.9831 & 1 & 0.4186 \\
\hline SRPX & $1.0079 \mathrm{E}-05$ & $3.2133 \mathrm{E}-08$ & 0.0078 & 0.0014 & $1.1851 \mathrm{E}-07$ & 1 & 0.0014 & $8.9930 \mathrm{E}-08$ \\
\hline
\end{tabular}

Bolded and underlined numbers represent significant $p$ values as derived from ${ }^{1}$ Mann-Whitney $U$ test, ${ }^{2}$ Kruskal-Wallis test, and ${ }^{3}$ Bonferroni-adjusted Dunn test 


\section{A}

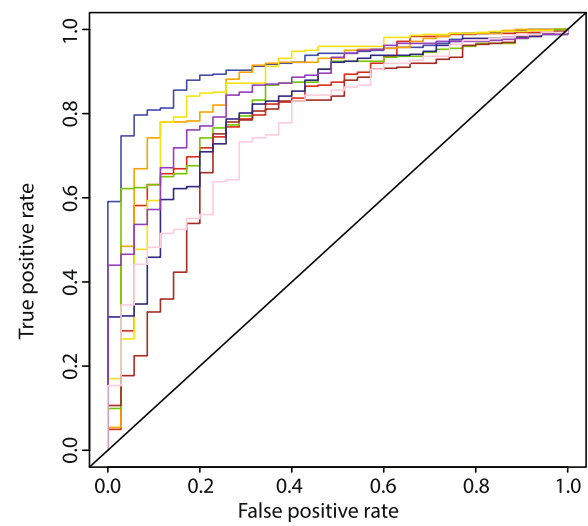

$\begin{array}{lll}\text { Gene } & \text { AUC } & \mathbf{9 5 \%} \mathbf{C l} \\ \text { ASB2 } & 0.828 & 0.756-0.900 \\ \text { ETNK2 } & 0.919 & 0.886-0.952 \\ \text { KCNJ15 } & 0.881 & 0.818-0.943 \\ \text { MEIS2 } & 0.876 & 0.808-0.944 \\ \text { NRG1 } & 0.841 & 0.779-0.903 \\ \text { NTN1 } & 0.769 & 0.683-0.856 \\ - \text { NUDT10 } & 0.821 & 0.751-0.890 \\ \text { PDE4A } & 0.861 & 0.806-0.916 \\ \text { SRPX } & 0.781 & 0.706-0.856\end{array}$

B
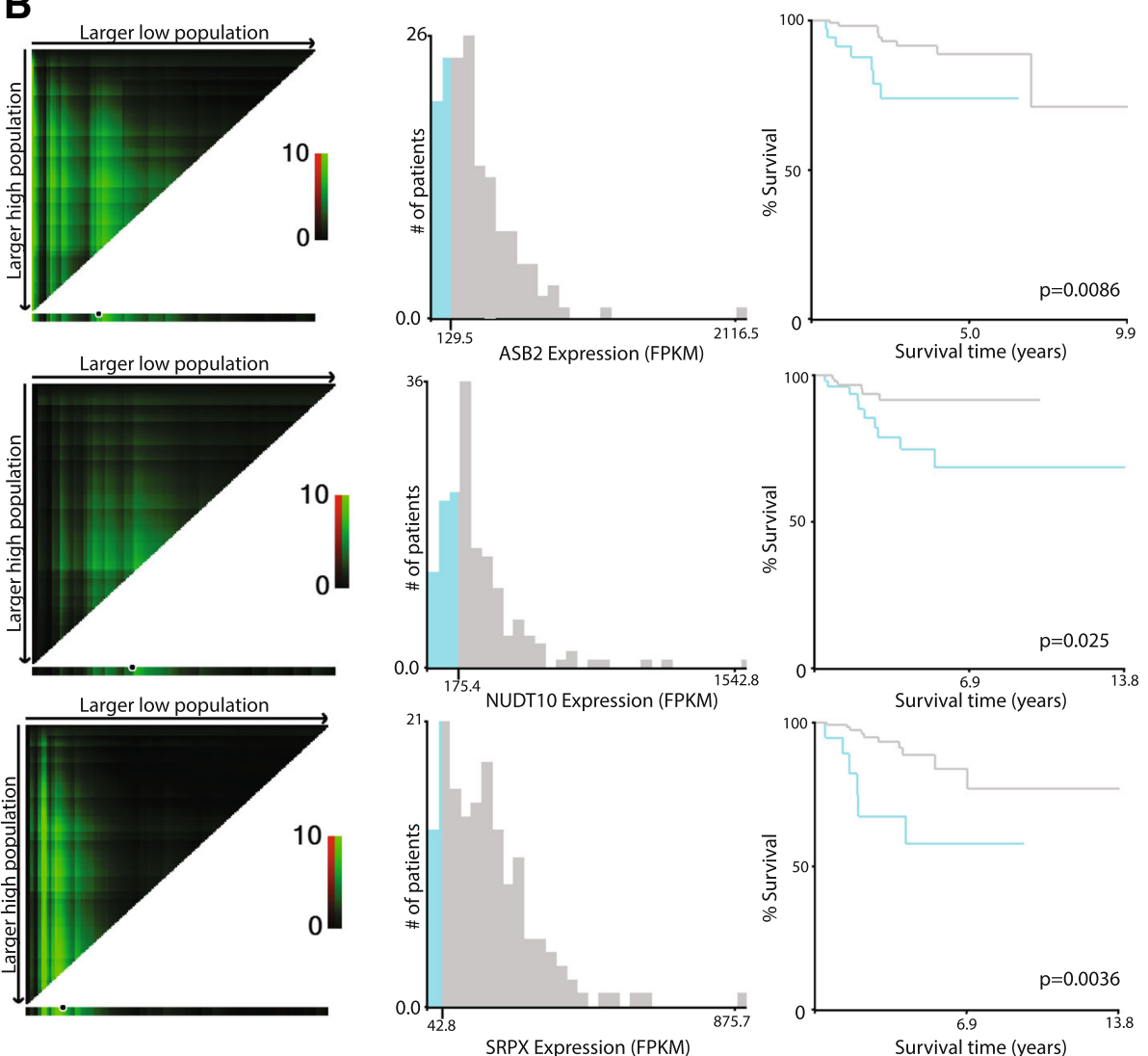

Fig. 4 Candidate gene expression is indicative of tumor status and can predict worse recurrence-free survival in patients. a Receiver operating characteristic (ROC) curves for individual candidate gene expression, stratifying between benign $(n=35)$ and tumor $(n=423)$ patients in the TCGA cohort. AUCs and 95\% confidence intervals for each gene are provided on the right. $\mathbf{b}$ X-tile analysis and Kaplan-Meier plots for prediction of biochemical recurrence-free survival in the TCGA cohort. Left: $X$-tile plots depict $x^{2}$ values for all possible data divisions, with brightness indicating strength of association and green coloration indicating a direct relationship. Black circles on the bottom bars for each graph depict automatically generated cut points maximizing the $x^{2}$ value in an auto-generated training set. Middle: Histogram depicting the number of patients in the auto-generated validation set below (blue) or above (gray) the cutoff point. Right: Kaplan-Meier plot showing recurrence-free survival in low-expressing (blue, below cutoff) or high-expressing (gray, above cutoff) groups for each gene. Log-rank $p$ values are indicated on each graph

Methylation of $S R P X$ was found to be completely independent of ERG fusion status in tumors. ASB2, MEIS2, and NRG1 methylation was increased in ERG-fusion positive tumors, while ETNK2, NTN1, and NUDT10 showed decreased methylation associated with ERG status (Additional file 13: Table S5). Gene probe methylation differences were validated in an independent methylation array dataset $(n=90$; accession number 
Table 2 Sensitivity and specificity for nine candidate genes in classifying prostate tumor vs normal

\begin{tabular}{lll}
\hline Gene & Sensitivity & Specificity \\
\hline ASB2 & 0.631 & 0.914 \\
ETNK2 & 0.797 & 0.943 \\
KCNJ15 & 0.778 & 0.886 \\
MEIS2 & 0.839 & 0.829 \\
NRG1 & 0.619 & 0.971 \\
NTN1 & 0.749 & 0.771 \\
NUDT10 & 0.787 & 0.743 \\
PDE4A & 0.759 & 0.829 \\
SRPX & 0.73 & 0.714 \\
\hline
\end{tabular}

GSE73549), with probes in all genes except for ASB2 able to significantly distinguish between normal tissues, prostate tumors, and lymph node $\mathrm{PCa}$ metastases (Kruskal-Wallis test $p<0.05$, Additional file 14: Table S6). Increased methylation of two probes in MEIS2 (cg01566404 and cg13800209) was also able to significantly distinguish between prostate tumor sites and metastatic cancer (Bonferroni-adjusted Dunn test, $p<0.05$ ).

All genes except $A S B 2$ exhibited significantly increased tumor methylation as compared to normal prostate in all significant proximal probes, while $A S B 2$ methylation was significantly decreased in TCGA prostate tumors at all significant probes within the promoter as well as its single proximal probe (Additional file 15: Figure S9, Table 5, Fig. 6). These findings were reflected in univariate logistic regression analyses of logistic-transformed methylation $\beta$-values ( $M$-values; Table 6$)$ in the entire TCGA dataset $(n=478)$, where ASB2 methylation probes were unique in exhibiting significant association with tumor status based on decreased, rather than increased, methylation values (ORs ranging from 0.09 to 0.30). Intriguingly, $A S B 2$ did not possess any probes located within the $A S B 2$ promoter methylation peak from the
TET2-KO differentially methylated site (DMS), raising the possibility that the increased methylation observed in TET2 $\mathrm{KO}$ cells was specific to that region. Most methylation probes exhibited strong discriminatory performance between tumor and normal tissue, with median AUC of 0.834 and probes within three genesMEIS2, NRG1, and SRPX-exhibiting sensitivities greater than $90 \%$ (Table 5). However, multivariate logistic regression analyses indicated that probes in ETNK2 $(\operatorname{cg} 20136584$, Wald $p$ value $=0.0014)$ and NRG1 $(\operatorname{cg} 00614182$, Wald $p$ value $=0.049)$ were independent predictors of tumor versus normal status in the TCGA cohort, indicating that increased methylation of these genes may be particularly important in PCa (Table 6).

Next, we examined methylation of these proximal probes in all tumors from the TCGA cohort $(n=428)$ as possible discriminators for the aforementioned clinicopathological variables (Table 4, Additional file 16: Figure S10). All seven genes with proximal probes possessed at least one probe able to significantly distinguish tumors of any GS $(6,7 \mathrm{a}$, $7 \mathrm{~b}$, or $8+$ ) from normal samples (Bonferroni-adjusted Dunn test, $p<0.05)$. Furthermore, all genes except MEIS2 and $A S B 2$ possessed at least one methylation probe able to significantly distinguish tumors based on recurrence (Mann-Whitney $U$ test, $p<0.05$ ). Kaplan-Meier analysis of these significant probes revealed that high methylation levels at three NRG1 probes were significantly associated with poor outcome in terms of recurrence-free survival (Fig. 5b). Two more probes in NRG1 (cg00614182, log-rank $p$ value $=0.0544)$ and ETNK2 (cg20136584, $\log$-rank $p$ value $=0.0693$ ) were trending for significance.

TET2-target gene ASB2 exhibits localized and specific gain of promoter methylation in prostate tumors within the TET2-target site

As $A S B 2$ was unique among the seven candidate genes in exhibiting a differential methylation pattern in its proximal methylation probes in the TCGA as compared

Table 3 Univariate and multivariate logistic regression analyses for classification of prostate tumor vs normal prostate

\begin{tabular}{lllllll}
\hline Gene & $\begin{array}{l}\text { Univariate } \\
p \text { value }\end{array}$ & $\begin{array}{l}\text { Univariate } \\
\text { odds ratio }\end{array}$ & $\begin{array}{l}\text { Univariate } \\
\text { confidence interval }\end{array}$ & $\begin{array}{l}\text { Multivariate } \\
p \text { value }\end{array}$ & $\begin{array}{l}\text { Multivariate } \\
\text { odds ratio }\end{array}$ & $\begin{array}{l}\text { Multivariate } \\
\text { confidence interval }\end{array}$ \\
\hline ASB2 & $2.05 \mathrm{E}-08^{* * *}$ & 0.998 & $0.997-0.998$ & 0.96 & 1 & $0.998-1.002$ \\
ETNK2 & $2.34 \mathrm{E}-13^{* * *}$ & 0.99 & $0.987-0.993$ & 0.1 & 0.996 & $0.992-1.001$ \\
KCNJ15 & $1.50 \mathrm{E}-10^{* * *}$ & 0.983 & $0.978-0.988$ & 0.17 & 0.995 & $0.988-1.002$ \\
MEIS2 & $7.25 \mathrm{E}-14^{* * *}$ & 0.997 & $0.997-0.998$ & $0.019^{*}$ & 0.998 & $0.997-1.000$ \\
NRG1 & $2.07 \mathrm{E}-08^{* * *}$ & 0.97 & $0.959-0.980$ & $0.028^{*}$ & 0.98 & $0.964-0.998$ \\
NTN1 & $2.08 \mathrm{E}-06^{* * *}$ & 0.997 & $0.995-0.998$ & $0.028^{*}$ & 1.004 & $1.000-1.007$ \\
NUDT10 & $1.26 \mathrm{E}-05^{* * *}$ & 0.998 & $0.997-0.999$ & $0.010^{*}$ & 0.999 & $0.998-1.000$ \\
PDE4A & $3.37 \mathrm{E}-10^{* * *}$ & 0.993 & $0.991-0.995$ & 0.064 & 0.997 & $0.993-1.000$ \\
SRPX & $1.42 \mathrm{E}-07^{* * *}$ & 0.994 & $0.992-0.997$ & 0.89 & 1 & $0.997-1.003$ \\
\hline
\end{tabular}

Statistical significance indicated by asterisks: ${ }^{*} 0.01<p<0.05 ;{ }^{* *} 0.001<p<0.01 ;{ }^{* * *} p<0.001$

Note: odds ratios are modest due to the large scale range of the data (see Fig. $4 b$ ) 


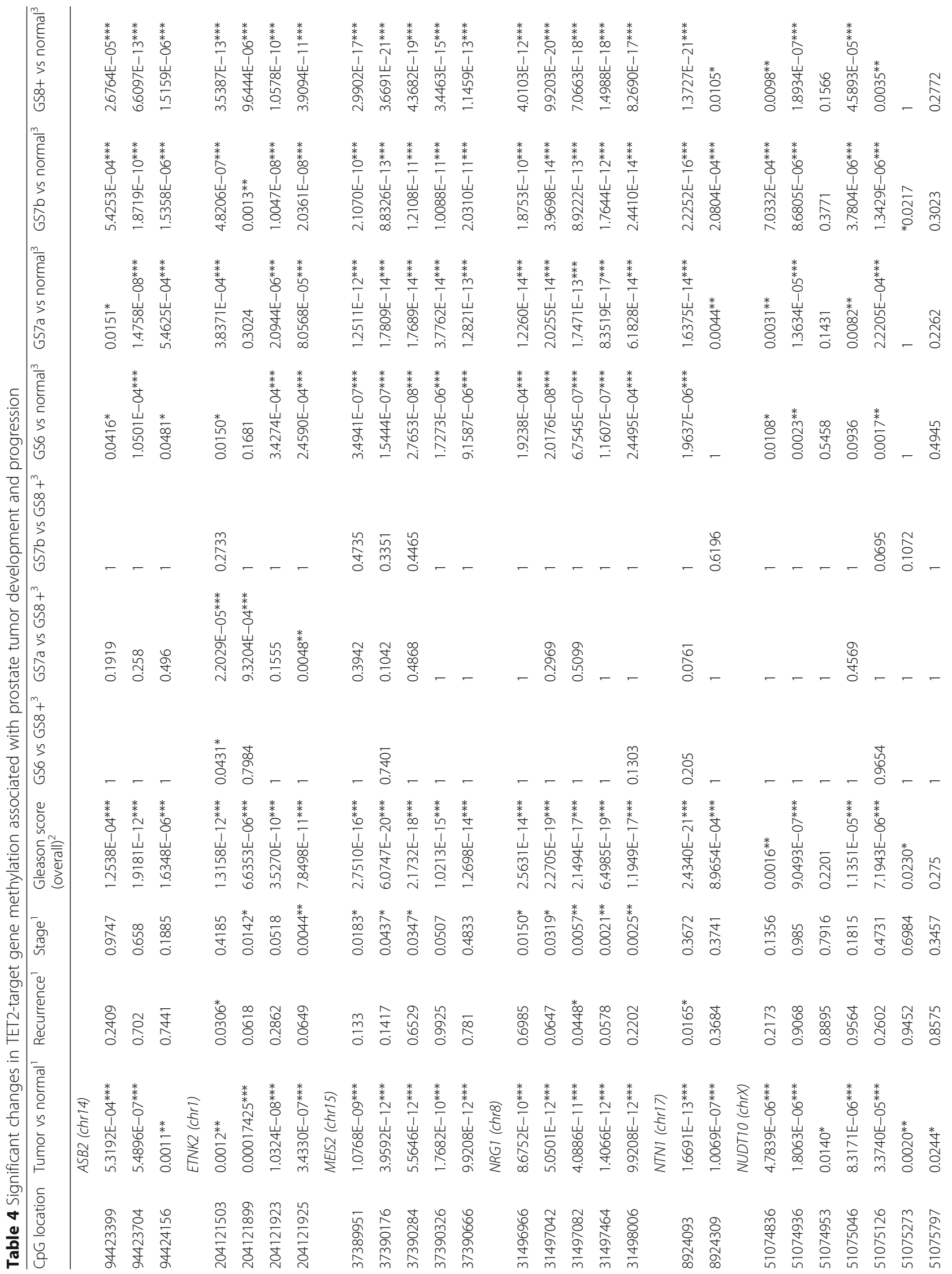




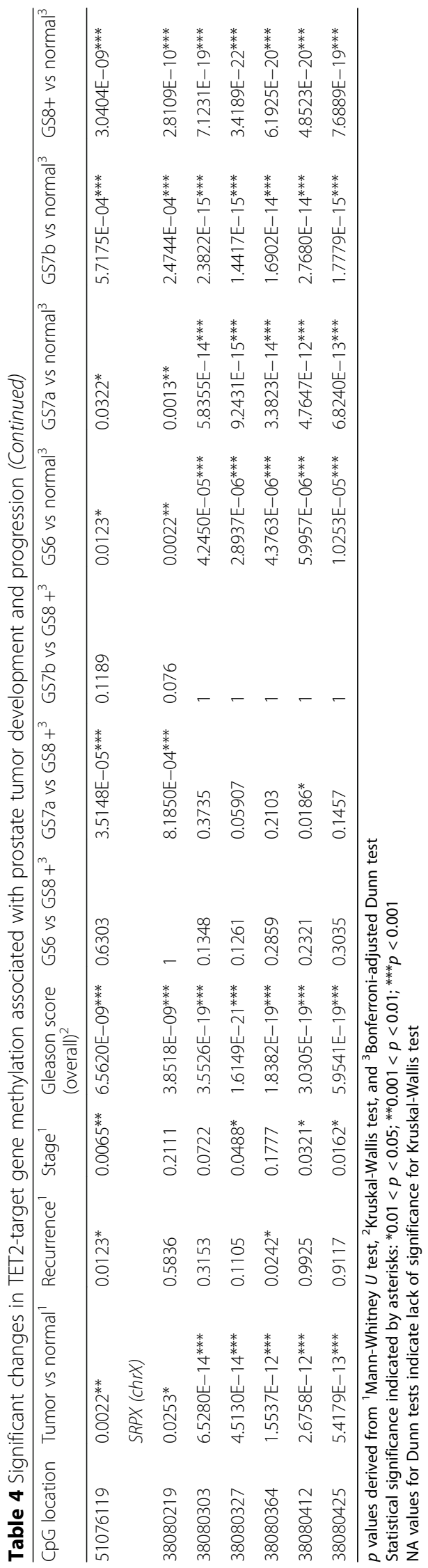




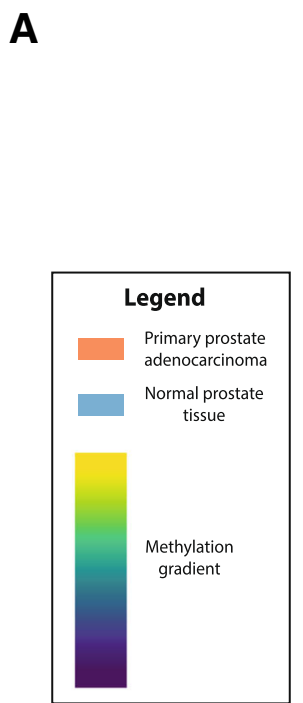

B
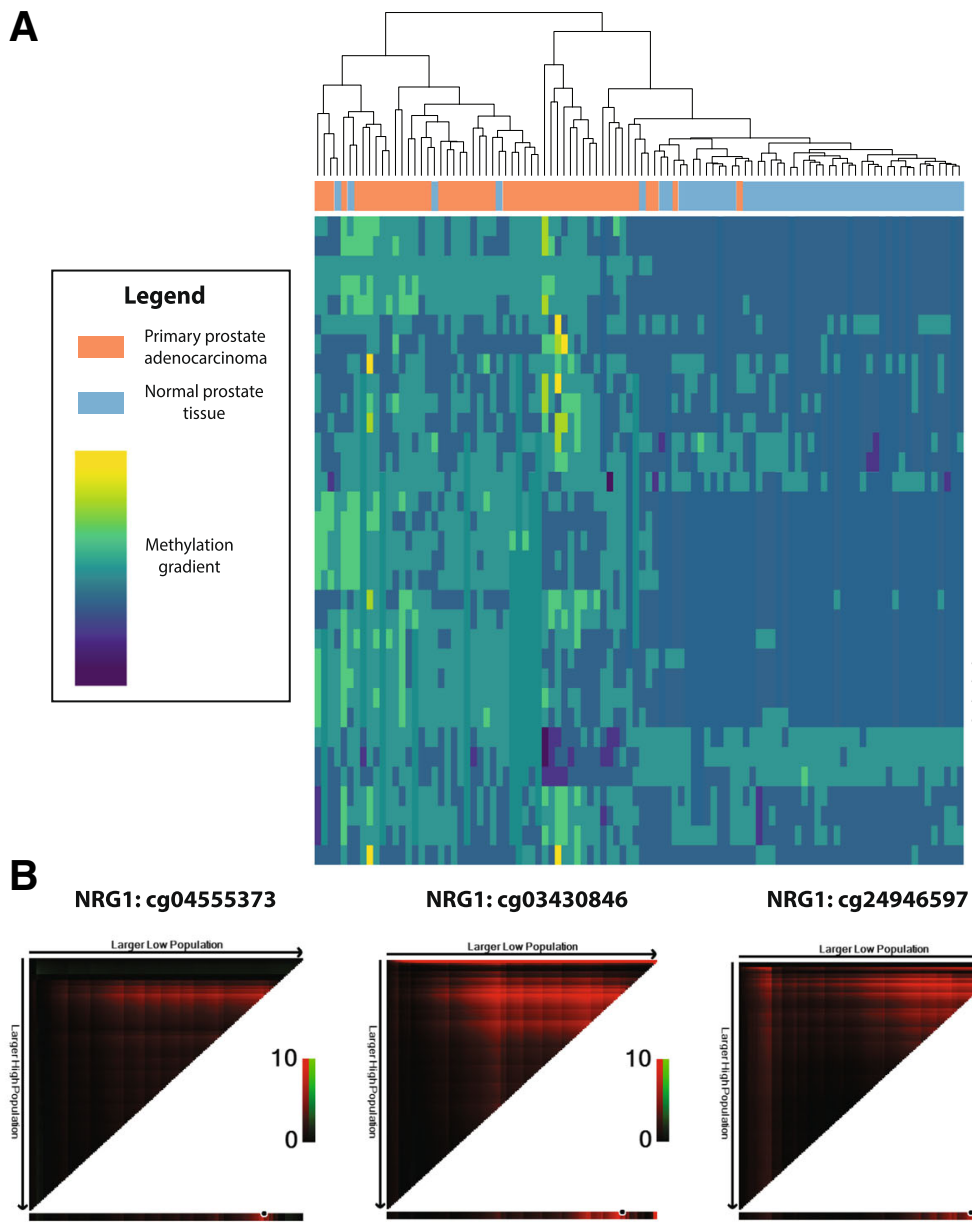

SRPX_cg03509565 SRPX_cg27485646 SRX_cg17407511
SRPX_cg19736094 SRPX_cg14759284 SRPX_cg16626088 NUDT10_cg09520583 NUDT10_cg06481089 NUDT10_cg20430749 NUDT10_cg02975846 NDDT_cg00648125 NUDT10_c 22363867 NUDT10_cg21844331 NRG1_cg00614182 NRG1_cg24946597 RRG1_cg03430846 NRG1_cg04555373 NRG1_cg04773818 NTN1_cg17072465 NTN1_cg14615768 MEIS2_Cg21643314 MEIS2_cg07433663 MEISL cg03951374 MEIS2_cg01958086 ASB2 cg09247392 ASB2_cg19949550 ASB2_cg01956154 ETNK2_Cg01566404 ETNK2_cg00103329 ETNK2_cg21535580
ETNK2_cg20136584

NRG1: cg03430846
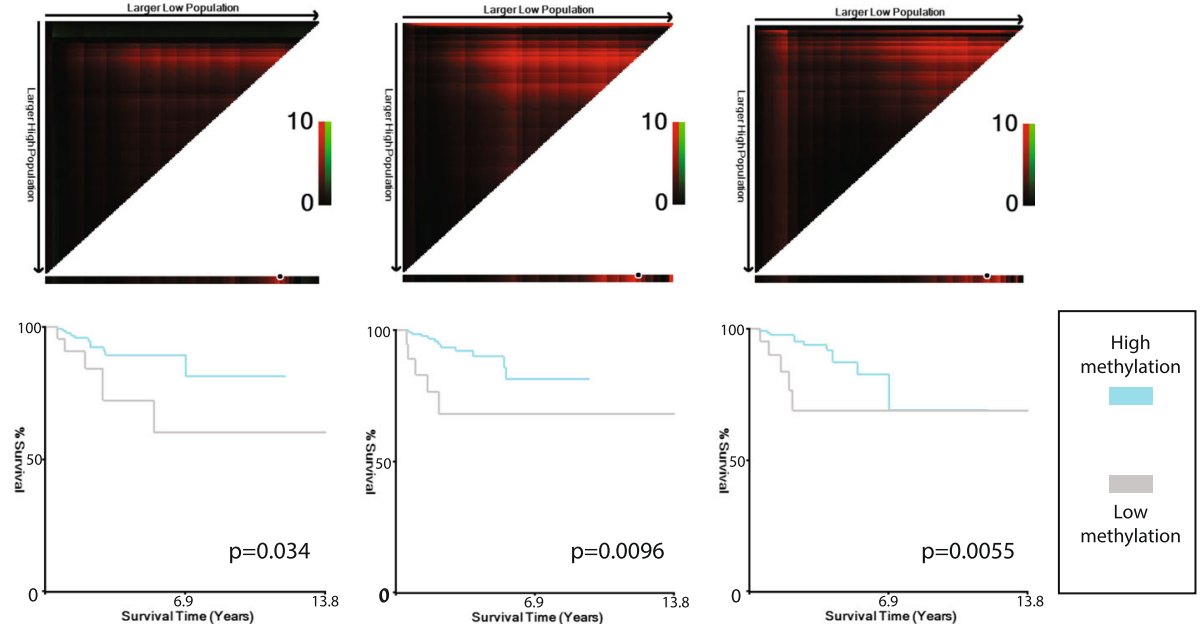

Fig. 5 Tumor methylation comparison of candidate genes and predictive ability for recurrence. a Unsupervised heatmap depicting methylation beta values normalized by probe for seven genes exhibiting significantly altered expression and methylation in both knockouts and matched prostate tumor and normal samples $(n=50)$. Methylation gradient bar indicates normalized methylation levels, ranging from highest (yellow) to lowest (dark blue). Dendrograms indicate clustering between tissue samples. $\mathbf{b}$ X-tile analysis depicting methylation probes significantly associated with outcome. High-methylation probe status (gray, above cutoff) was indicative of worse recurrence-free survival as compared to patients with low-methylation probe status (blue, below cutoff) for the three probes shown. Log-rank $p$ values are indicated on each graph

to our KOs, we selected this gene for further validation to assess the specificity and accuracy of the TET2-directed methylation changes observed in our cell line model.

Following validation of ASB2 methylation and expression data from sequencing via MBD-qPCR and RT-qPCR, respectively, in both $\mathrm{KO}$ and parental cells (Additional file 17: Figure S11 and Additional file 18: Figure S12), we examined methylation levels of our TET2-KO-specific DMR in an independent, limited series of primary patient samples comprised of matched normal and tumor tissues ( $n=19$ per group).

We found that, in concordance with the results observed in our TET2 $\mathrm{KO}$ models and in contrast to our observations in the TCGA dataset, methylation of the $A S B 2$ promoter region was significantly increased in tumor samples as compared to normal $(p=0.04067$, paired Wilcoxon signed-rank test, Fig. 7). This observation underscores both qualitative and quantitative 
Table 5 Sensitivity and specificity of candidate gene methylation in classifying prostate tumor vs normal prostate tissue

\begin{tabular}{|c|c|c|c|c|}
\hline Gene & Probe ID & Sensitivity & Specificity & $A \cup C$ \\
\hline ASB2 & cg01956154 & 0.514 & 0.8 & 0.684 \\
\hline ASB2 & cg19949550 & 0.759 & 0.8 & 0.834 \\
\hline ASB2 & cg09247392 & 0.61 & 0.84 & 0.747 \\
\hline ETNK2 & cg20136584 & 0.61 & 0.92 & 0.803 \\
\hline ETNK2 & cg21535580 & 0.486 & 0.9 & 0.687 \\
\hline ETNK2 & cg00103329 & 0.612 & 0.94 & 0.798 \\
\hline ETNK2 & cg01566404 & 0.703 & 0.86 & 0.79 \\
\hline MEIS2 & cg01958086 & 0.811 & 0.84 & 0.879 \\
\hline MEIS2 & cg03951374 & 0.86 & 0.92 & 0.917 \\
\hline MEIS2 & cg13800209 & 0.914 & 0.84 & 0.909 \\
\hline MEIS2 & cg07433663 & 0.79 & 0.88 & 0.881 \\
\hline MEIS2 & cg21643314 & 0.867 & 0.82 & 0.871 \\
\hline NRG1 & cg04773818 & 0.794 & 0.9 & 0.849 \\
\hline NRG1 & cg04555373 & 0.902 & 0.88 & 0.912 \\
\hline NRG1 & cg03430846 & 0.888 & 0.86 & 0.891 \\
\hline NRG1 & cg24946597 & 0.855 & 0.92 & 0.914 \\
\hline NRG1 & cg00614182 & 0.862 & 0.84 & 0.881 \\
\hline NTN1 & cg14615768 & 0.839 & 0.94 & 0.926 \\
\hline NTN1 & cg17072465 & 0.481 & 0.94 & 0.671 \\
\hline NUDT10 & cg21844331 & 0.381 & 0.86 & 0.62 \\
\hline NUDT10 & cg22363867 & 0.755 & 0.7 & 0.747 \\
\hline NUDT10 & cg15159291 & 0.341 & 0.86 & 0.61 \\
\hline NUDT10 & cg00648125 & 0.558 & 0.84 & 0.712 \\
\hline NUDT10 & cg02975846 & 0.558 & 0.88 & 0.705 \\
\hline NUDT10 & cg20430749 & 0.311 & 1 & 0.558 \\
\hline NUDT10 & cg06481089 & 0.306 & 0.92 & 0.603 \\
\hline NUDT10 & cg09520583 & 0.5 & 0.92 & 0.75 \\
\hline SRPX & cg16626088 & 0.762 & 0.66 & 0.771 \\
\hline SRPX & cg14759284 & 0.818 & 0.92 & 0.902 \\
\hline SRPX & cg19736094 & 0.895 & 0.9 & 0.925 \\
\hline SRPX & $\operatorname{cg} 17407511$ & 0.874 & 0.9 & 0.909 \\
\hline SRPX & cg27485646 & 0.902 & 0.84 & 0.905 \\
\hline SRPX & cg03509565 & 0.8 & 0.92 & 0.905 \\
\hline
\end{tabular}

differences occurring in methylation marks within the TET2-targeted ASB2 gene region.

\section{Discussion}

Multiple lines of evidence have implicated a role for dysregulation of the master epigenetic regulator TET2 in PCa development and progression. Although TET2 is less frequently mutated in primary $\mathrm{PCa}$ as compared to metastatic PCa, several factors have been hypothesized as potentially contributing to its loss of expression, including hypoxia, which deprives TET enzymes of the oxygen required for their dioxygenase activity, alterations in expression of TET-governing transcription factors such as high-mobility group AT-hook 2 (HGMA2), and repression of TET by oncogenic miRNAs [25, 28]. Furthermore, TET2 exhibits high mutation rates (10-20\%) and extensive loss of heterozygosity ( $60 \%)$ in metastatic prostate tumors [21-23, 28]. Genome-wide association studies have also shown increased PCa risk linked to an intergenic TET2-proximal SNP (rs7679673) $[23,29]$. However, perhaps due to its low somatic mutation rates in primary prostate cancer, TET2-mediated changes have not been systematically investigated as potential drivers of cancer development. Therefore, we performed TET2 $\mathrm{KO}$ in normal prostate RWPE-1 cells to identify targets of TET2 mediation that may be important in prostate carcinogenesis.

Of the two different KOs, CR1 (the heterozygous knockout) showed unique methylation targeting of 487 genes, while the homozygous knockout model CR2 had 2682 uniquely methylated genes. TET2 haploinsufficiency is enough to alter cellular properties and contribute to hematological malignancies, possibly due to a decrease in its catalytic activity [26, 30]. However, as complete loss of TET2 protein is observed in both KOs, these findings suggest that certain TET2 mutations may cause a dominant negative phenotype. The usage of alternative compensatory mechanisms (such as increased activity of other TET enzymes) may explain the differential profiles between the two KOs; however, further studies are required to determine whether the type of TET2 mutation influences the use of alternative $5 \mathrm{hmC}$ pathways.

Interestingly, we found that biological pathways affected by methylation gain in TET2 KOs overlapped with intergenic $5 \mathrm{hmC}$ gain that we previously reported in PCa cell lines [16]. Paradoxically, despite the extensive global loss of $5 \mathrm{hmC}$ in $\mathrm{PCa}$, increases in intergenic $5 \mathrm{hmC}$ levels were found to occur in genes related to inflammation and cellular adhesion-the same pathways enriched among silenced genes from our knockout model-resulting in downregulation of immune and adhesive functions. These observations suggest that not only are these critical functions governed by TET2 dysregulation, but also that intergenic $5 \mathrm{hmC}$ and promoter $5 \mathrm{mC}$ gain may work synergistically to downregulate the same genes and pathways in cancer.

Intriguingly, out of seven key TET2-governed genes identified from our model exhibiting differential methylation in normal versus tumor samples in the TCGA at TET2-KO DMS-proximal probes, only one-ASB2showed significantly decreased, rather than increased, methylation levels in cancer. However, the other target genes possessed probes either within the peaks identified through our methylation sequencing strategy (ETNK2, MEIS2, NRG1, NUDT10) or closer to these DMSs than 


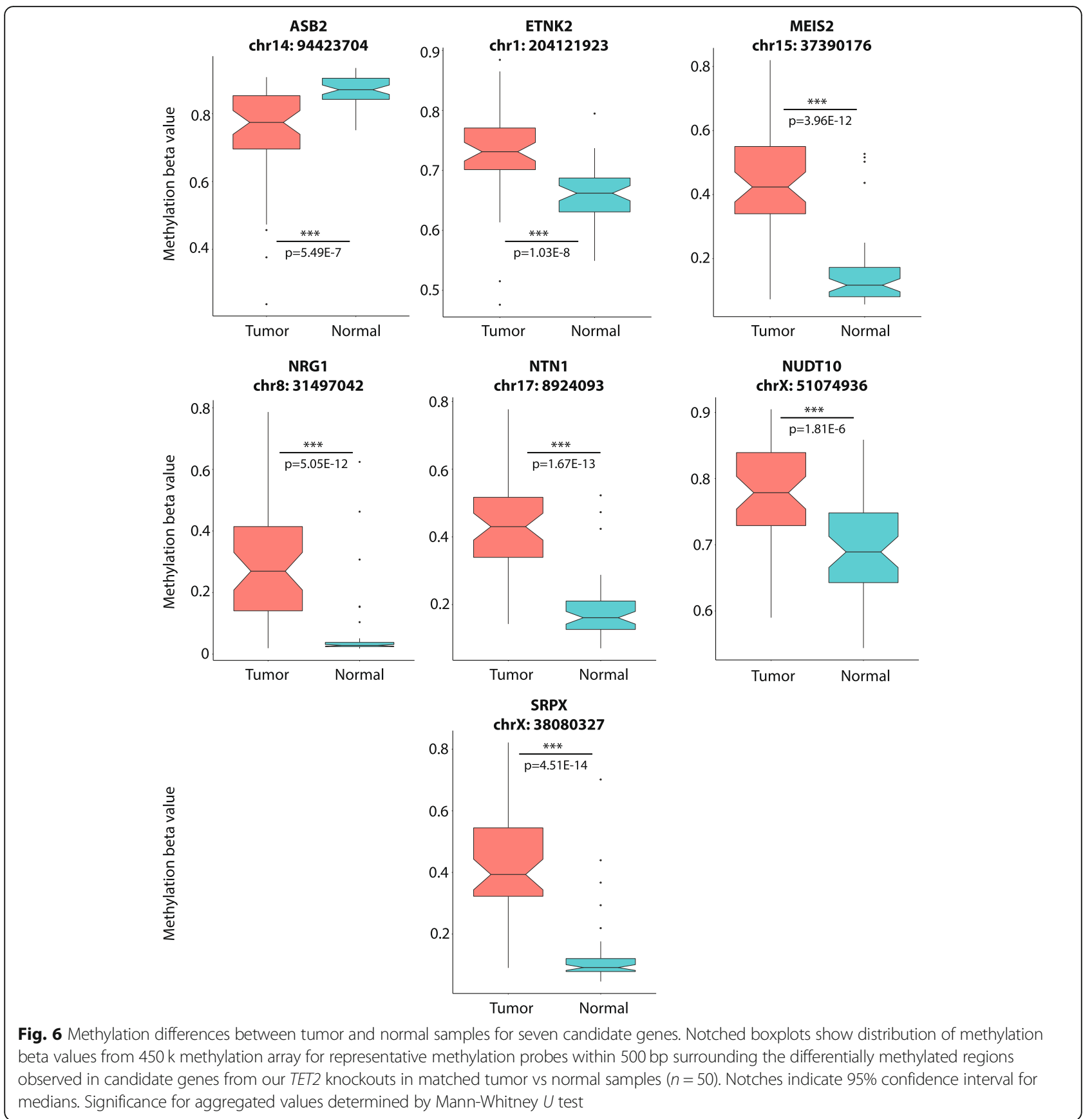

those for $A S B 2$ (at distances of $328 \mathrm{bp}, 160 \mathrm{bp}$, or $22 \mathrm{bp}$ for $A S B 2, N T N 1$, or $S R P X$, respectively), and examination of methylation in matched tumor and normal samples from an independent series indicated concordant and increased $5 \mathrm{mC}$ within the ASB2 TET2-KO DMS. Furthermore, TET2-KO differentially methylated sites in all candidate genes were highly conserved in primates, ranging from $71.4 \%(5 / 7)$ of CpGs exhibiting conservation among at least three primate species in $A S B 2$ to $100 \%$ conservation for all CpGs in ETNK2, MEIS2, NRG1, and SRPX. This sequence conservation suggests that these TET2-mediated regions may possess a functionally important role.

Methylation of ETNK2, NTN1, and NUDT10 was increased in ERG-fusion negative tumors, which is concordant with the significant loss of TET2 expression in these tumor samples. The increased methylation of MEIS2 and NRG1 in ERG-positive tumors indicates that TMPRSS2-ERG fusion impacts the methylation status of these genes. However, expression of all seven candidate genes was also found to be independent of ERG fusion status in prostate tumors, which may indicate that their 
Table 6 Univariate and multivariate logistic regression analyses for classification of prostate tumor vs normal prostate based on probe methylation

\begin{tabular}{|c|c|c|c|c|c|c|c|}
\hline Gene & Probe & $\begin{array}{l}\text { Univariate } \\
p \text { value }\end{array}$ & $\begin{array}{l}\text { Univariate } \\
\text { odds ratio }\end{array}$ & $\begin{array}{l}95 \% \text { confidence } \\
\text { interval }\end{array}$ & $\begin{array}{l}\text { Multivariate } \\
p \text { value }\end{array}$ & $\begin{array}{l}\text { Multivariate } \\
\text { odds ratio }\end{array}$ & $\begin{array}{l}95 \% \text { confidence } \\
\text { interval }\end{array}$ \\
\hline ASB2 & cg01956154 & $3.48 \mathrm{E}-05^{* * *}$ & 0.3 & $0.17-0.53$ & 0.72 & 1.46 & $0.18-11.84$ \\
\hline ASB2 & cg19949550 & $5.37 \mathrm{E}-11^{* * *}$ & 0.18 & $0.11-0.30$ & 0.09 & 0.3 & $0.070-1.20$ \\
\hline ASB2 & cg09247392 & $4.42 \mathrm{E}-07^{* * *}$ & 0.09 & $0.04-0.24$ & 0.18 & 0.12 & $0.010-2.60$ \\
\hline ETNK2 & cg20136584 & $4.89 \mathrm{E}-09^{* * *}$ & 16.64 & $6.49-42.68$ & ${ }^{* *} 0.0014$ & 154.88 & $6.95-3449.78$ \\
\hline ETNK2 & cg21535580 & $6.27 \mathrm{E}-05^{* * *}$ & 3.17 & $1.80-5.59$ & 0.08 & 19.76 & $0.70-559.54$ \\
\hline ETNK2 & cg00103329 & $1.09 \mathrm{E}-08^{* * *}$ & 8.27 & $4.01-17.07$ & 0.26 & 11.95 & $0.15-933.7$ \\
\hline ETNK2 & cg01566404 & $1.38 \mathrm{E}-08^{* * *}$ & 5.69 & $3.12-10.36$ & 0.17 & 0.09 & $0.00-2.78$ \\
\hline MEIS2 & cg01958086 & $1.12 \mathrm{E}-14^{* * *}$ & 2.85 & 2.19-3.72 & 0.73 & 0.81 & $0.24-2.70$ \\
\hline MEIS2 & cg03951374 & $<2.00 \mathrm{E}-16^{* * *}$ & 3.88 & $2.84-5.29$ & 0.77 & 0.81 & $0.20-3.33$ \\
\hline MEIS2 & cg13800209 & $2.08 \mathrm{E}-12^{* * *}$ & 2.57 & $1.97-3.34$ & 0.064 & 1.88 & $0.96-3.66$ \\
\hline MEIS2 & cg07433663 & $<2.00 \mathrm{E}-16^{* * *}$ & 2.35 & $1.92-2.88$ & 0.46 & 0.75 & $0.35-1.60$ \\
\hline MEIS2 & cg21643314 & $8.79 \mathrm{E}-13^{* * *}$ & 3.77 & $2.62-5.43$ & 0.71 & 0.79 & $0.23-2.72$ \\
\hline NRG1 & cg04773818 & $3.41 \mathrm{E}-14^{* * *}$ & 2.08 & $1.72-2.52$ & 0.67 & 0.85 & $0.40-1.80$ \\
\hline NRG1 & cg04555373 & $3.96 \mathrm{E}-14^{* * *}$ & 3.18 & $2.35-4.28$ & 0.071 & 4.67 & $0.88-24.94$ \\
\hline NRG1 & cg03430846 & $2.84 \mathrm{E}-14^{* * *}$ & 3.5 & $2.54-4.84$ & 0.31 & 0.44 & $0.090-2.11$ \\
\hline NRG1 & cg24946597 & $2.41 \mathrm{E}-11^{* * *}$ & 2.82 & $2.08-3.83$ & 0.81 & 0.9 & $0.37-2.16$ \\
\hline NRG1 & cg00614182 & $1.16 \mathrm{E}-13^{* * *}$ & 3.76 & $2.65-5.34$ & ${ }^{*} 0.049$ & 2.7 & $1.70-26.00$ \\
\hline NTN1 & $\operatorname{cg} 14615768$ & $<2.00 \mathrm{E}-16^{* * *}$ & 6.12 & $4.03-9.27$ & 0.46 & 0.53 & $0.10-2.83$ \\
\hline NTN1 & cg17072465 & $0.000213^{* * *}$ & 3.25 & $1.74-6.08$ & 0.36 & 0.32 & $0.030-3.71$ \\
\hline NUDT10 & cg21844331 & 0.14 & 1.37 & $0.90-2.09$ & NA & NA & NA \\
\hline NUDT10 & cg22363867 & $4.44 \mathrm{E}-07^{* * *}$ & 3.28 & $2.07-5.21$ & 0.3 & 0.37 & $0.060-2.41$ \\
\hline NUDT10 & cg15159291 & $0.02^{*}$ & 2.82 & $1.18-6.78$ & 0.35 & 0.18 & $0.00-6.51$ \\
\hline NUDT10 & cg00648125 & $4.38 \mathrm{E}-05^{* * *}$ & 2.94 & $1.75-4.93$ & 0.9 & 0.91 & $0.21-3.98$ \\
\hline NUDT10 & cg02975846 & $2.53 \mathrm{E}-05^{* * *}$ & 2.7 & $1.70-4.29$ & 0.19 & 3.84 & $0.52-28.62$ \\
\hline NUDT10 & cg20430749 & $0.023^{*}$ & 1.51 & $1.06-2.15$ & 0.23 & 0.32 & $0.050-2.05$ \\
\hline NUDT10 & cg06481089 & 0.13 & 0.65 & $0.37-1.13$ & NA & NA & NA \\
\hline NUDT10 & cg09520583 & $6.25 E-06^{* * *}$ & 6.47 & $2.88-14.55$ & 0.2 & 2.72 & $0.58-12.76$ \\
\hline SRPX & $\operatorname{cg} 16626088$ & $2.13 \mathrm{E}-08^{* * *}$ & 26.69 & $8.46-84.23$ & 0.99 & 1.01 & $0.090-11.53$ \\
\hline SRPX & cg14759284 & $4.31 \mathrm{E}-13^{* * *}$ & 6 & $3.70-9.74$ & 0.98 & 0.99 & $0.30-3.24$ \\
\hline SRPX & cg19736094 & $6.00 \mathrm{E}-13^{* * *}$ & 5.11 & $3.44-7.58$ & 0.57 & 0.62 & $0.12-3.14$ \\
\hline SRPX & $\operatorname{cg} 17407511$ & $<2.00 \mathrm{E}-16^{* * *}$ & 4.82 & $3.36-6.91$ & 0.38 & 2.11 & $0.39-11.28$ \\
\hline SRPX & cg27485646 & $1.03 \mathrm{E}-11^{* * *}$ & 6.98 & $3.99-12.23$ & 0.56 & 0.66 & $0.16-2.66$ \\
\hline SRPX & cg03509565 & $8.40 \mathrm{E}-13^{* *}$ & 8.04 & $4.54-14.23$ & 0.073 & 5.25 & $0.85-32.27$ \\
\hline
\end{tabular}

Odds ratios and $p$ values presented for methylation $M$ values (logistic-transformed beta values)

Statistical significance indicated by asterisks: ${ }^{*} 0.01<p<0.05$; ${ }^{* *} p<0.001$

expression is influenced more strongly by TET2 status than by mutation subtype in PCa. Taken together, these findings suggest that TET2 loss results in highly localized region-specific, rather than widespread, changes in methylation at conserved regions within target gene promoters, which may have profound effects on gene expression.

Several prior studies have indicated the functional importance of our candidate gene panel in cancer development and progression. All candidate genes except ethanolamine kinase 2 (ETNK2) have been specifically linked to $\mathrm{PCa}$ in some manner. The cell migration-critical gene netrin 1 (NTN1), cellular adhesion molecule neuregulin 1 (NRG1), and the putative tumor suppressor sushi repeat-containing protein X-linked (SRPX) exhibit downregulation in prostate cancer as compared to normal prostate or benign prostate hyperplasia tissue [30-33], and evidence for association between the expression of ubiquitin ligase $A S B 2$ and $\mathrm{PCa}$ progression is established in the 


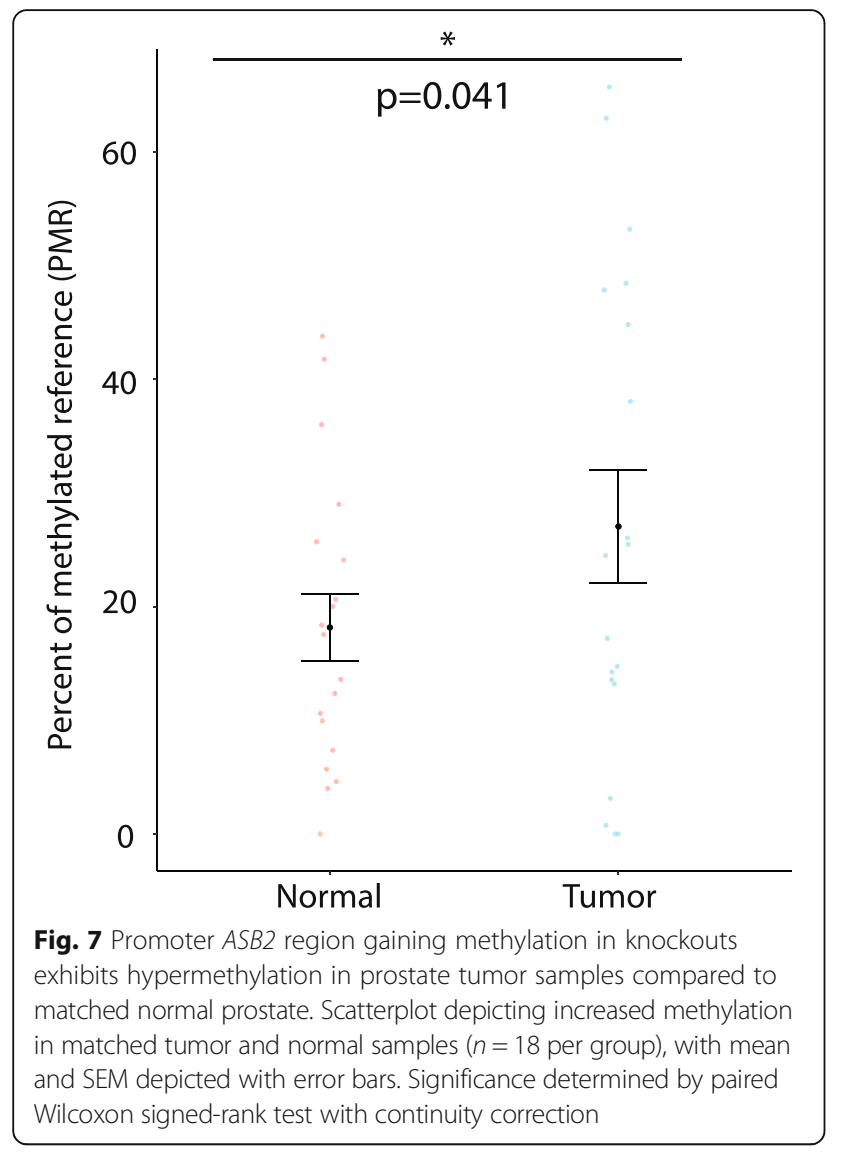

literature [34, 35]. Intriguingly, although we found NRG1 probe methylation status to be significantly associated with recurrence-free survival by Kaplan-Meier analyses, its expression was not, even though both NRG1 expression and methylation were associated with TET2 expression status. This may indicate that candidate gene expression may be influenced in a methylation-independent manner (such as TET2-mediated O-GlcNAcylation); however, further studies are required to elucidate the mechanisms of this relationship.

Loss of the transcriptional regulator MEIS2 is not only associated with recurrence and worse survival in $\mathrm{PCa}$ patients [36], but is also associated with the development of castration-resistant prostate cancer due to its inhibition resulting in constitutive activation of nuclear factor kappa-light-chain-enhancer of activated B cells (NF-kB) signaling [37]. Although expression of nudix hydrolase 10 (NUDT10) has not yet been examined in the context of $\mathrm{PCa}$, the rs5945572 risk SNP within this gene is significantly associated with increased risk of $\mathrm{PCa}$ in Caucasian, African, and Asian ethnic groups, functionally implicating this gene in prostate cancer [38]. Intriguingly, several of these genes are upregulated in other types of cancers, including gastric, ovarian, and breast cancers, indicating that the mechanisms by which they contribute to $\mathrm{PCa}$ may be unique. Overall, further exploration of the TET2 KO DMRs within the target genes identified by this study may uncover new insight into the mechanisms of carcinogenic changes in PCa.

In conclusion, we have demonstrated the utility of our TET2-knockout based candidate selection strategy in identifying significantly altered genes and potential markers of prostate carcinogenesis. Correlation of TET2-target genes with clinicopathological characteristics of PCa revealed a panel of seven promising candidates-ASB2, ETNK2, MEIS2, NRG1, NTN1, NUDT10, and $S R P X$-exhibiting discriminatory ability between tumor and normal samples and/or measures of PCa progression based on their $5 \mathrm{mC}$ and expression status. Lowered expression of three of these genes (ASB2, NUDT10, and $S R P X)$, as well as increased methylation of NRG1, was significantly correlated with lower recurrence-free survival in PCa patients, showing the utility of these TET2 targets as potential markers of disease. Validation of one such target gene, $A S B 2$, indicated high specificity of TET2-mediated methylation, which may provide insight into the functionality of TET2-directed changes in PCa. Ultimately, future biological studies of epigenetic and transcriptomic disruption of candidate gene in cancer may allow us to not only identify new diagnostic and prognostic markers for $\mathrm{PCa}$, but may also provide novel insights into the dynamic changes underlying development and progression in many different cancers exhibiting TET2 dysregulation.

\section{Supplementary information}

Supplementary information is available at Clinical Epigenetics' website.

\section{Materials and methods \\ Cell culture and CRISPR knockout}

Normal human prostate epithelial cell line, RWPE-1, was obtained from the American Type Culture Collection (ATCC, Manassas, Virginia). For CRISPR knockout, cells were transfected using Lipofectamine as per the manufacturer's protocol with commercially available Sigma (St. Louis, Missouri) CRISPR plasmid expressing gRNA targeting the first coding exon of TET2, as well as Cas9 fused to GFP via a 2A linker peptide (gRNA target ID: HS0000114238; sequence: TTAGTAGCCTGACT GTTAA with TGG protospacer-associated motif). Forty-eight hours post-transfection, GFP-FACS was used to perform single-cell sorting of successfully transfected cells onto 96-well plates. Post-expansion, populations were assayed via Sanger sequencing (The Centre for Applied Genomics, Toronto) for the presence of indels. Off-target analysis was performed as described by Mali et al. [27]. RWPE-1 and knockout cells were cultured with keratinocyte serum-free medium (K-SFM) 
(Invitrogen, catalog \#17005042), supplemented with $0.05 \mathrm{mg} / \mathrm{mL}$ bovine pituitary extract (BPE) and $5 \mathrm{ng} / \mathrm{mL}$ human recombinant epidermal growth factor (EGF). All cells were cultured as a monolayer and maintained in a humidified incubator at $37^{\circ} \mathrm{C}$ with $5 \% \mathrm{CO}_{2}$.

\section{Patient cohort}

Radical prostatectomy tissue samples used in this study were comprised of treatment-naïve patients with $\mathrm{PCa}$ with matched normal tissue samples and were accrued for gene methylation analysis as per REB guidelines. Gleason score 7 samples were collected from October 2002 to July 2007, with a median follow-up time of 5.04 years (ranging from 3.88 to 9.79 years). Full clinical information for these patients can be found in Additional file 19: Table S7.

\section{DNA and RNA extraction}

DNA was extracted from cell pellets using the QIAamp DNA Mini Kit (Qiagen, catalog \#51306) following the manufacturer's protocol. Whole RNA was extracted via TRIzol method as per the manufacturer's protocol (Thermo Fisher, catalog \#15596026) and was cleaned up using the RNeasy Mini Kit (Qiagen, catalog \#74104).

\section{Whole-methylome and whole-transcriptome sequencing: MBD-Seq and RNA-Seq}

$2.5 \mu \mathrm{g}$ sheared genomic DNA from RWPE-1 and CR1/CR2 knockout cells was enriched for $5 \mathrm{mC}$ with the MethylMiner kit (Invitrogen, catalog \#ME10025) as previously described [16]. Enriched DNA was submitted in duplicate along with unenriched input controls for library preparation (NEBNext ${ }^{\circ}$ ChIP-seq Library Prep Reagent Set for Illumina) and high-throughput sequencing using the HiSeq 2500 (Illumina, San Diego, California) at The Centre for Applied Genomics. Each library generated approximately 75 million paired-end reads. RNA library preparation for each of the above cell lines was performed following the NEB NEBNext Ultra Directional Library Preparation protocol (poly-A mRNA) on 200 ng total RNA. Libraries were sequenced in duplicate using the HiSeq 2500 at The Centre for Applied Genomics, with each library generating approximately 30 million paired-end reads.

\section{Bioinformatics analysis of MBD-Seq}

Trimmed reads were mapped to hg19 using Bowtie2 (v2.2.1). [39] Repitools (v 1.12.1) was used to evaluate bound, enriched sample enrichment compared to input (Additional file 20: Figure S13). Significantly enriched regions of methylation were identified using Model-based Analysis of ChIP-Seq algorithm (MACS v 2.0.10). [40] DiffBind (v 1.12.2) was used to derive consensus peaks based on the presence of a peak in at least one sample analyzed. Annotation was performed using ChIPpeakAnno R package (v.2.16.4) [41] and a customized version of Annovar program [42] with RefSeq genes to determine specific genomic features closest to the peaks.

\section{Bioinformatics analysis of RNA-Seq}

Trimmed reads were aligned to the hg19 human genome using Tophat v2.0.11, and raw read counts were extracted using htseq-count v0.6.1p2, with only uniquely mapping reads being counted. Raw gene counts were then loaded and sample-normalized using DESeq v1.18.0, and MDS analysis was performed to determine sample separation (Additional file 21: Figure S14). Two-condition differential expression was done with edgeR v3.8.6, filtering for only genes that were expressed. Quality of raw and trimmed reads was assessed with FastQC v0.11.2 and FastQ-Screen v0.4.3, and read distribution and strandedness was assessed with RSeQC v2.3.7. The above two analyses were performed by The Centre for Applied Genomics (Toronto).

\section{Western blotting}

Cells were lysed with RIPA buffer supplemented with protease inhibitors and incubated on ice for $10 \mathrm{~min}$, then centrifuged at $14,000 \mathrm{RPM}$ for $10 \mathrm{~min}$ at $4{ }^{\circ} \mathrm{C}$. Fifty-microgram samples with SDS loading dye were boiled and run on a 7.5\% SDS-PAGE gel. Proteins were detected by immunoblotting (using 5\% milk in TBS-T as blocking reagent) with antibodies against TET2 (Abcam, ab94580) or Ku80 (Cell Signaling Technologies, C48E7) overnight at $4{ }^{\circ} \mathrm{C}$ Unedited Western blot images from Figure $1 \mathrm{~B}$ are shown in Additional 22: Figure S15.

\section{qRT-PCR}

Primers were designed for specific targeting of ASB2-001 (isoform 2) and ASB2-002 (isoform 1) (GrCh37) so that the shared reverse primer spanned the junctions between exons 4 and 5 in isoform 1 and exons 3 and 4 in isoform 2. The forward primer for isoform 1 was located in its second exon (which is unique to isoform 1), while the forward primer for isoform 2 was located within a 67-bp region in its first exon unique to isoform 2 (Additional file 2: Table S1). Reverse transcription was performed using the qScript cDNA SuperMix kit (Quantabio, catalog \#95048-100), and expression levels of transcripts were analyzed using SYBR Green qPCR.

\section{Pathway analysis of MBD-Seq datasets correlated with RNA-Seq expression data}

Promoter region was defined as $-1500 \mathrm{bp}$ to $+500 \mathrm{bp}$ from the transcription start site of a given gene. Promoter regions gaining methylation and losing expression in knockouts compared to RWPE-1 were listed, and pathway enrichment analysis was performed using the Genomic Regions Enrichment of Annotations Tool (GREAT). [43] The whole human genome was used as background. 


\section{MethyLight}

Genomic DNA from matched normal and tumors was bisulfite converted and subjected to the MethyLight assay as previously described [44], using $A L U$ as a methylation reference (Additional file 2: Table S1). Each sample was analyzed in duplicate on the Applied Biosystems $^{\oplus} 7500$ Real-Time PCR System. Methylation levels were determined using the delta-delta $\mathrm{Ct}$ method, with supermethylated (CH3) DNA from EMD Millipore (catalog \#S7821) as control. Genes with Ct for ALU exceeding a value of 30 were excluded from the analysis.

\section{Statistical analyses}

Association between candidate gene expression or methylation and tumor versus normal status or outcome was analyzed using Kruskal-Wallis and Mann-Whitney $U$ tests as part of the base "stats" package of $\mathrm{R}$ (v3.4.0). Bonferroni correction was applied by dividing 0.05 by the number of samples analyzed and using the resultant value as the confidence threshold. Receiver operator curve (ROC) analysis was used to assess the discriminatory ability of candidate gene expression between tumor and normal samples. ROC plots, confidence intervals (via DeLong's test), and AUC values were generated using the ROCR package in R (v1.0.7). Sensitivity and specificity were calculated based on the identification of the optimal cutoff point from ROC curve analysis maximizing both values. Univariate and multivariate logistic regression analyses were performed for individual gene expression values via generalized linear model analysis in $\mathrm{R}$, using Wald $p$ values to determine significance, and odds ratios were calculated via the logistic display function of the epiDisplay package (v3.5.0.1).

X-tile software (v3.6.1) was used to split the TCGA dataset into two parts, and X-tile plots were used to determine the optimal cutoff point for prediction of biochemical recurrence in the training set (cutoff points generated automatically by X-tile software) [45]. This cutoff was applied to the validation set as generated by $\mathrm{X}$-tile software, and Kaplan-Meier survival curves and $\log$-rank $p$ values were used to assess individual gene performance. $p<0.05$ was used as the confidence threshold for the above analyses unless otherwise specified in the manuscript.

\section{Additional files}

Additional file 1: Figure S1. CRISPR-TET2 guide RNA targets the first coding exon of TET2. Arrows indicate the CRISPR target site on gene diagram of the functional TET2 isoform 1, which truncates the protein before any functional domains are produced. (TIF $134 \mathrm{~kb}$ )

Additional file 2: Table S1. Primer and probe sequences used for CRISPR verification and methylation-specific GPCR. (XLSX $17 \mathrm{~kb}$ )

Additional file 3: Figure S2. Off-target analysis of CRISPR-TET2 clones (CR1 and CR2). Sanger sequencing chromatograms for the top two gene regions partially matching the CRISPR-TET2 gRNA sequence shows no off-target effects of CRISPR on the parental gene sequence for either CR1 (top) or CR2 (bottom). (TIF 7908 kb)

Additional file 4: Figure S3. Overexposed Western blot for TET2 protein in parental RWPE-1 and TET2-KO cells. Overexposed Western blot shows no detectable bands for either TET2 isoform for CR1 or CR2 knockouts as compared to parental RWPE-1 cells. (TIF 4414 kb)

Additional file 5: Figure S4. Heatmap showing methylation of all genes exhibiting increased promoter methylation in TET2-KOs as compared to RWPE-1 cells. Methylation gradient bar indicates genenormalized methylation levels, ranging from highest (yellow) to lowest (dark blue). Heatmap was generated via unsupervised clustering and clusters RWPE-1 cells separately from knockouts. (TIF 29540 kb)

Additional file 6: Figure S5. Heatmap showing expression of all differentially expressed and downregulated genes in both TET2-KOs as compared to RWPE-1 cells. Expression gradient bar indicates gene-normalized expression levels, ranging from highest (yellow) to lowest (dark blue). Heatmap was generated via unsupervised clustering and clusters RWPE-1 cells separately from knockouts. (TIF $29524 \mathrm{~kb}$ )

Additional file 7: Table S2. Genes exhibiting both significantly increased promoter methylation and significantly lowered expression in CRISPR TET2-KO cells. (XLSX $26 \mathrm{~kb}$ )

Additional file 8: Figure S6. Heatmap showing expression of 780 genes which exhibited significant expression alterations in knockout cells as well as low-TET2 expressing tumors, for all tumor $(n=423)$ and normal $(n=35)$ samples in the TCGA. Expression gradient bar indicates gene-normalized expression levels, ranging from highest (yellow) to lowest (dark blue). Heatmap was generated via unsupervised clustering. (TIF 36633 kb)

Additional file 9: Table S3. Characteristics of the 61 genes exhibiting significantly increased promoter methylation and lowered expression in TET2-KOs as well as significantly lowered expression in TCGA prostate tumors. (XLSX $18 \mathrm{~kb}$ )

Additional file 10: Table S4. Expression of candidate genes significantly associated with ERG fusion status. (XLSX $18 \mathrm{~kb}$ )

Additional file 11: Figure S7. Pathway enrichment analysis of genes losing expression in CRISPR-TET2 knockout cells. Visual depiction of key pathways identified from GREAT analysis of all genes silenced in both CR1 and CR2, using the whole genome as a background. (TIF $917 \mathrm{~kb}$ )

Additional file 12: Figure S8. X-tile analysis and Kaplan-Meier plots for prediction of biochemical recurrence-free survival in the TCGA cohort. Left: $X$-tile plots depict $X^{2}$ values for all possible data divisions, with brightness indicating strength of association and green coloration indicating a direct relationship. Black circles on the bottom bars for each graph depict automatically generated cut points maximizing the $x^{2}$ value in an auto-generated training set. Middle: Histogram depicting the number of patients in the auto-generated validation set below (blue) or above (gray) the cutoff point. Right: Kaplan-Meier plot showing recurrence-free survival in low-expressing (blue; below cutoff) or high-expressing (gray; above cutoff) groups for each gene. Log-rank $p$ values are indicated on each graph. (EPS 3514 kb)

Additional file 13: Table S5. Association of candidate gene probe methylation with ERG fusion status. (XLSX 19 kb)

Additional file 14: Table S6. Analysis of candidate gene probes in an independent methylation array dataset (GSE73549). (XLSX 20 kb)

Additional file 15: Figure S9. Methylation array probe locations on candidate genes with respect to the TET2-knockout methylated site and transcription start sites. Numbers indicate the genomic location of each probe (blue circle), transcription start site (green triangle), or TET2-knockout differentially methylated site (red rectangle). Probes chosen are within 500 base pairs of TET2-KO sites except for ASB2, which includes two additional probes within the promoter region. (TIF $37076 \mathrm{~kb}$ )

Additional file 16: Figure S10. Heatmap showing methylation of seven candidate genes for all tumor $(n=428)$ and normal $(n=50)$ samples in the TCGA. Methylation gradient bar indicates gene-normalized methylation beta values, ranging from highest (yellow) to lowest (dark blue). Heatmap was generated via unsupervised clustering. (TIF 33437 kb) 
Additional file 17: Figure S11. Methylation of ASB2 is increased in both knockouts (CR1 and CR2) within the TET2-knockout cell differentially methylated site. Expression analysis performed using MethyLight methylation-specific qPCR $(n=3)$. (TIF $472 \mathrm{~kb})$

Additional file 18: Figure S12. Expression of both ASB2 isoforms is lowered in both knockouts as compared to parental RWPE-1 cells. Expression analysis performed using qRT-PCR $(n=3)$. (TIF $1636 \mathrm{~kb})$

Additional file 19: Table S7. Clinicopathological characteristics of prostate tumor tissues used for methylation analysis. (XLSX $15 \mathrm{~kb}$ )

Additional file 20: Figure S13. Diagnostic enrichment plot of MBD-Seq samples from TET2-KO cells as compared to input controls. Enrichment diagnostic graphs comparing the curve of input (non-enriched) sample (blue and yellow lines) to biological replicates of CR1 (green and orange lines) and CR2 (purple and red lines), respectively. (TIF $12551 \mathrm{~kb}$ )

Additional file 21: Figure S14. Multidimensional scaling (MDS) plot depicting Euclidean distances (similarities) between RNA-sequencing samples. MDS plot shows relative similarities between RWPE-1, TET2-KO cells, and the prostate adenocarcinoma 22Rv1 cell line based on RNA sequencing results. (TIF $13562 \mathrm{~kb}$ )

Additional file 22: Proteins were detected by immunoblotting with antibodies. (TIF $118 \mathrm{~kb}$ )

\section{Acknowledgements}

Not applicable

\section{Funding}

This work was supported by Prostate Cancer Canada and the Movember Foundation under Translation Acceleration Grant number 2014-01. SNK was funded by the Ontario Graduate Scholarship (OGS) - Faculty of Medicine at University of Toronto (UofT), Scace Graduate Fellowship in Prostate Cancer Research (UofT), Frank Fletcher Memorial Fund (UofT), and the Peterborough K.M. Hunter OSOTF Award (UofT).

\section{Availability of data and materials}

The data sets supporting the results of this article will be made available in the Gene Expression Omnibus (GEO) repository following acceptance, under accession number GSE128399 (The Cancer Genome Atlas datasets analysed in this study are publicly available online at https://portal.gdc.cancer.gov. [5]

\section{Authors' contributions}

SNK carried out CRISPR knockout cell line generation, $5 \mathrm{mC}$ enrichment, and sample preparation/submission for sequencing; performed and analyzed all data including integrative analysis and pathway analysis, candidate gene panel identification, heatmap generation/interpretation, statistical analyses, MethyLight, and $\mathrm{gPCR}$ experiments; and drafted the manuscript. RH and GB helped with pathway analysis and interpretation. AZ, TvdK, and $\mathrm{NH}$ participated in the design, coordination, and critical review of the manuscript. BB conceived of the study, participated in its design and coordination, and helped to draft the manuscript. All authors read and approved the final manuscript.

\section{Ethics approval and consent to participate}

Matched human prostate tumor and normal tissue was obtained as per ethical approvals established by the Research Ethics Board of Mount Sinai Hospital, Toronto, Ontario (REB reference \#140071-E).

\section{Consent for publication}

Not applicable

\section{Competing interests}

The authors declare that they have no competing interests.

\section{Publisher's Note}

Springer Nature remains neutral with regard to jurisdictional claims in published maps and institutional affiliations.

\section{Author details}

'Lunenfeld-Tanenbaum Research Institute, Mount Sinai Hospital, 60 Murray Street, L6-304B, Toronto, ON M5T 3L9, Canada. ${ }^{2}$ Department of Laboratory
Medicine and Pathobiology, University of Toronto, Medical Sciences Building (6th floor), 1 King's College Circle, Toronto, ON M5S 1A8, Canada. ${ }^{3}$ Terrence Donnelly Centre for Cellular and Biomolecular Research, University of Toronto, 160 College St, Toronto, ON M5S 3E1, Canada. ${ }^{4}$ Department of Pathology, University Health Network, University of Toronto, 200 Elizabeth St, Toronto, ON M5G 2C4, Canada. ${ }^{5}$ Department of Surgery and Surgical Oncology, Division of Urology, University Health Network, University of Toronto, 190 Elizabeth St, Toronto, ON M5G 2C4, Canada.

Received: 18 December 2018 Accepted: 10 March 2019

Published online: 27 March 2019

\section{References}

1. Ferlay J, Soerjomataram I, Dikshit R, Eser S, Mathers C, Rebelo M, et al. Cancer incidence and mortality worldwide: sources, methods and major patterns in GLOBOCAN 2012. Int J Cancer. 2015;136(5):E359-86.

2. Wong MCS, Goggins WB, Wang HH, Fung FD, Leung C, Wong SY, et al. Global incidence and mortality for prostate cancer: analysis of temporal patterns and trends in 36 countries. Eur Urol. 2016;70(5):862-74.

3. Foley R, Hollywood D, Lawler M. Molecular pathology of prostate cancer: the key to identifying new biomarkers of disease. Endocr Relat Cancer. 2004; 11(3):477-88.

4. Fraser M, Sabelnykova VY, Yamaguchi TN, Heisler LE, Livingstone J, Huang V, et al. Genomic hallmarks of localized, non-indolent prostate cancer. Nature. 2017;541(7637):359-64

5. Cancer Genome Atlas Research Network. The molecular taxonomy of primary prostate cancer. Cell. 2015;163(4):1011-25.

6. Packer JR, Maitland NJ. The molecular and cellular origin of human prostate cancer. Biochim Biophys Acta. 2016;1863(6 Pt A):1238-60.

7. Jerónimo C, Henrique R, Hoque MO, Mambo E, Ribeiro FR, Varzim G, et al. A quantitative promoter methylation profile of prostate cancer. Clin Cancer Res. 2004;10(24):8472-8.

8. Pan J, Chen J, Zhang B, Chen X, Huang B, Zhuang J, et al. Association between RASSF1A promoter methylation and prostate cancer: a systematic review and meta-analysis. PLoS One. 2013;8(9):e75283.

9. Cairns P, Esteller M, Herman JG, Schoenberg M, Jeronimo C, SanchezCespedes M, et al. Molecular detection of prostate cancer in urine by GSTP1 hypermethylation. Clin Cancer Res. 2001;7(9):2727-30.

10. Jerónimo C, Usadel H, Henrique R, Silva C, Oliveira J, Lopes C, et al. Quantitative GSTP1 hypermethylation in bodily fluids of patients with prostate cancer. Urology. 2002;60(6):1131-5.

11. Lee WH, Isaacs WB, Bova GS, Nelson WG. CG island methylation changes near the GSTP1 gene in prostatic carcinoma cells detected using the polymerase chain reaction: a new prostate cancer biomarker. Cancer Epidemiol Biomark Prev. 1997;6(6):443-50.

12. Zhao F, Olkhov-Mitsel E, van der Kwast T, Sykes J, Zdravic D, Venkateswaran $V$, et al. Urinary DNA methylation biomarkers for noninvasive prediction of aggressive disease in patients with prostate cancer on active surveillance. J Urol. 2017;197(2):335-41.

13. Liu L, Kron KJ, Pethe W, Demetrashvili N, Nesbitt ME, Trachtenberg J, et al. Association of tissue promoter methylation levels of APC, TGF $32, \mathrm{HOXD} 3$ and RASSF1A with prostate cancer progression. Int J Cancer. 2011;129(10):2454-62.

14. Olkhov-Mitsel E, van der Kwast T, Kron K, Ozcelik H, Briollais L, Massey C, et al. Quantitative DNA methylation analysis of genes coding for kallikreinrelated peptidases 6 and 10 as biomarkers for prostate cancer. Epigenetics. 2012;7(9):1037-45

15. Strand SH, Hoyer S, Lynnerup AS, Haldrup C, Storebjerg TM, Borre M, et al. High levels of 5-hydroxymethylcytosine (5hmC) is an adverse predictor of biochemical recurrence after prostatectomy in ERG-negative prostate cancer. Clin Epigenetics. 2015;7:111.

16. Kamdar $\mathrm{SN}$, Ho LT, Kron $\mathrm{K}$, Isserlin $\mathrm{R}$, van der Kwast $\mathrm{T}$, Zlotta $\mathrm{AR}$, et al. Dynamic interplay between locus-specific DNA methylation and hydroxymethylation regulates distinct biological pathways in prostate carcinogenesis. Clin Epigenetics. 2016;8:32

17. Pastor WA, Aravind L, Rao A. TETonic shift: biological roles of TET proteins in DNA demethylation and transcription. Nat Rev Mol Cell Biol. 2013;14(6):341-56.

18. Rasmussen KD, Helin K. Role of TET enzymes in DNA methylation, development, and cancer. Genes Dev. 2016;30:733-50.

19. Yang H, Liu Y, Bai F, Zhang JY, Ma SH, Liu J, et al. Tumor development is associated with decrease of TET gene expression and 5-methylcytosine hydroxylation. Oncogene. 2013;32(5):663-9. 
20. Takayama K, Misawa A, Suzuki T, Takagi K, Hayashizaki Y, Fujimura T, et al. TET2 repression by androgen hormone regulates globa hydroxymethylation status and prostate cancer progression. Nat Commun. 2015:6:8219.

21. Jeschke J, Collignon E, Fuks F. Portraits of TET-mediated DNA hydroxymethylation in cancer. Curr Opin Genet Dev. 2016:36:16-26.

22. Nickerson L, Im KM, Misner KJ, Tan W, Lou H, Gold B, et al. Somatic alterations contributing to metastasis of a castration resistant prostate cancer. Hum Mutat. 2013;34(9):1231-41.

23. Nickerson ML, Das S, Im KM, Turan S, Berndt S, Li H, et al. TET2 binds the androgen receptor and loss is associated with prostate cancer. Oncogene. 2017;36(15):2172-83.

24. Smeets E, Lynch AG, Prekovic S, Van den Broeck T, Moris L, Helsen C, et al. The role of TET-mediated DNA hydroxymethylation in prostate cancer. Mol Cell Endocrinol. 2017. https://doi.org/10.1016/j.mce.2017.08.021.

25. Dhiman VK, Attwood K, Campbell MJ, Smiraglia DJ. Hormone stimulation of androgen receptor mediates dynamic changes in DNA methylation patterns at regulatory elements. Oncotarget. 2015;6(40): 42575-89.

26. Ko M, Huang Y, Jankowska AM, Pape UJ, Tahiliani M, Bandukwala HS, et al. Impaired hydroxylation of 5-methylcytosine in myeloid cancers with mutant TET2. Nature. 2010;468:839-43.

27. Mali P, Esvelt KM, Church GM. Cas9 as a versatile tool for engineering biology. Nat Methods. 2013;10(10):957-63.

28. Huang Y, Rao A. Connections between TET proteins and aberrant DNA modification in cancer. Trends Genet. 2014;30(10):464-74.

29. Choudhury AD, Eeles R, Freedland SJ, Isaacs WB, Pomerantz MM, Schalken $J A$, et al. The role of genetic markers in the management of prostate cancer. Eur Urol. 2012;62(4):577-87.

30. Latil A, Chêne L, Cochant-Priollet B, Mangin P, Fournier G, Berthon $P$, et al. Quantification of netrins, slits and their receptors in human prostate tumors. Int J Cancer. 2003;103(3):306-15.

31. Latil A, Bièche I, Chêne L, Laurendeau I, Berthon P, Cussenot O, et al. Gene expression profiling in clinically localized prostate cancer: a four-gene expression model predicts clinical behavior. Clin Cancer Res. 2003;9(15):5477-85.

32. Kim CJ, Shimakage M, Kushima R, Mukaisho K, Shinka T, Okada Y, et al. Down-regulation of drs mRNA in human prostate carcinomas. Hum Pathol. 2003;34(7):654-7.

33. Stamey TA, Warrington JA, Caldwell MC, Chen Z, Fan Z, Mahadevappa M, et al. Molecular genetic profiling of Gleason grade $4 / 5$ prostate cancers compared to benign prostatic hyperplasia. J Urol. 2001;166(6):2171-7.

34. Erho N, Buerki C, Triche TJ, Davicioni E, Vergara IA. Transcriptome-wide detection of differentially expressed coding and non-coding transcripts and their clinical significance in prostate cancer. J Oncol. 2012. https://doi.org/ 10.1155/2012/541353.

35. Yu YP, Landsittel D, Jing L, Nelson J, Ren B, Liu L, et al. Gene expression alterations in prostate cancer predicting tumor aggression and preceding development of malignancy. J Clin Oncol. 2004;22:2790-9.

36. Chen JL, Li J, Kiriluk KJ, Rosen AM, Paner GP, Antic T, et al. Deregulation of a Hox protein regulatory network spanning prostate cancer initiation and progression. Clin Cancer Res. 2012;18(16):4291-302.

37. Jeong JH, Park SJ, Dickinson SI, Luo JL. A constitutive intrinsic inflammatory signaling circuit composed of miR-196b, Meis2, PPP3CC, and p65 drives prostate cancer castration resistance. Mol Cell. 2017;65(1):154-67.

38. Wencheng L, Gu M. NUDT11 rs5945572 polymorphism and prostate cancer risk: a meta-analysis. Int J Clin Exp Med. 2015;8(3):3474-81.

39. Langmead B, Trapnell C, Pop M, Salzberg SL. Ultrafast and memory-efficient alignment of short DNA sequences to the human genome. Genome Biol. 2009. https://doi.org/10.1186/gb-2009-10-3-r25.

40. Zhang Y, Liu T, Meyer CA, Eeckhoute J, Johnson DS, Bernstein BE, et al. Model-based analysis of ChIP-Seq (MACS). Genome Biol. 2008. https://doi. org/10.1186/gb-2008-9-9-r137.

41. Zhu L, Gazin C, Lawson ND, Pagès H, Lin SM, Lapointe DS, et al. ChIPpeakAnno: a Bioconductor package to annotate ChIP-seq and ChIP-chip data. BMC Bioinformatics. 2010. https://doi.org/10.1186/1471-2105-11-237.

42. Wang $\mathrm{K}$, Li M, Hakonarson H. ANNOVAR: functional annotation of genetic variants from high-throughput sequencing data. Nucleic Acids Res. 2010. https://doi.org/10.1093/nar/gkq603.

43. McLean CY, Bristor D, Hiller M, Clarke SL, Schaar BT, Lowe CB, et al. GREAT improves functional interpretation of cis-regulatory regions. Nat Biotechnol. 2010. https://doi.org/10.1038/nbt.1630.
44. Kron KJ, Liu L, Pethe W, Demetrashvili N, Nesbitt ME, Trachtenberg J, et al. DNA methylation of HOXD3 as a marker of prostate cancer progression. Lab Investig. 2010;90:1060-7.

45. Camp RL, Dolled-Filhart M, Rimm DL. X-tile: a new bioinformatics tool for biomarker assessment and outcome-based cut-point optimization. Clin Cancer Res. 2004. https://doi.org/10.1158/1078-0432.CCR-04-0713.
Ready to submit your research? Choose BMC and benefit from:

- fast, convenient online submission

- thorough peer review by experienced researchers in your field

- rapid publication on acceptance

- support for research data, including large and complex data types

- gold Open Access which fosters wider collaboration and increased citations

- maximum visibility for your research: over $100 \mathrm{M}$ website views per year

At $\mathrm{BMC}$, research is always in progress.

Learn more biomedcentral.com/submissions 